\title{
IDENTIFICATION OF HARDENING PARAMETERS USING FINITE ELEMENT MODELS AND FULL-FIELD MEASUREMENTS: SOME CASES STUDY
}

\author{
Laurent ROBERT ${ }^{*}$, Vincent VELAY, Nicolas DECULTOT and Souleymane RAMDE \\ Université de Toulouse; Mines Albi; ICA (Institut Clément Ader); \\ Campus Jarlard, F-81013 Albi cedex 09, France
}

\begin{abstract}
:
This paper is focused on the identification of elasto-plastic behaviour parameters of aluminium alloy 2024-T3 using full-field measurements. An orthotropic Hill criterion including an isotropic hardening is considered. Standard tensile tests provide a first set of parameters. Some of these parameters are optimised thanks to an inverse modelling including finite element analysis and experimental full-field displacement measurements. This so-called Finite Element Model Updated (FEMU) process is based on different specimen geometries which induced heterogeneous strain fields. Full-field measurements are provided by Digital Image Correlation (DIC) technique. The aim of this work is to study the impact of the specimen shape that gives the heterogeneous strain fields on the identification of isotropic hardening parameters. Results are compared and discussed.
\end{abstract}

\section{Keywords:}

plasticity, parameter identification, inverse problem, full-field measurement, digital image correlation, stereocorrelation, displacement field, strain field, finite element analysis, heterogeneity

Running head title: FEM Updating: some cases study

\footnotetext{
*Fax: +33 5634932 42, Email: laurent.robert@ mines-albi.fr
} 


\section{INTRODUCTION}

This paper is focused on the elasto-plastic behaviour parameters identification of aluminium sheet that are commonly used in many industrial forming processes like stamping or deep drawing. For that purpose, a methodology was developed to determine multiaxial material properties. It is based on a mixed numerical and experimental approach using Finite Element Model (FEM) results and optical full-field measurements. Today, full-field measurements methods are increasingly used in experimental mechanics [1-3]. They allow to measure kinematic fields (displacement or strain) and provide extended possibilities to identify multiaxial behaviour models from a single more complex mechanical test. Indeed the number of unknown parameters which characterises anisotropic elasto-plastic materials is quite large and their identification leads to several mechanical tests. For instance, at least three uniaxial tensile tests at different angles from the rolling direction $\left(0^{\circ}, 45^{\circ}\right.$ and $\left.90^{\circ}\right)$ are needed to identify the parameters of the most common anisotropic criterion [4]. A bulge test is also needed for more accurate criterion $[5,6]$. It is worth pointing out that this kind of identification approach based on full-field kinematic measurements allows releasing some experimental constraints of the standard mechanical tests (e.g. necessity of homogeneous test, perfect geometries, controlled boundary conditions, etc.).

Several behaviour parameters identification strategies based on full-field measurements have been developed recently [7], namely the equilibrium gap method [8], the constitutive equation error method [9], the virtual field method [10-12] and the Finite Element Model Updated (FEMU) method [13-19] considered in this work. The finite element method is generally used to obtain the numerical solution of the direct problem by providing displacement/strain/stress fields knowing loading conditions, specimen geometry and constitutive equations. It can be also used as a tool for solving iteratively the inverse problem, leading to the so-called mixed experimental/numerical methods. In this case, a first calculation is performed with an initial set of constitutive parameters. Nodal displacements are collected and compared to their experimental counterparts. The difference is quantified with an objective 
function, which is often the sum of the squared differences between numerical and experimental data. The idea is then to minimise iteratively this estimator with respect to the constitutive parameters. Many optimisation algorithms are available for minimising this objective function. They are often based on the numerical calculation of a sensitivity matrix [18], which allows the step-by-step determination of new sets of constitutive parameters. The procedure stops when the objective function is lower than a given threshold value. Such procedures have been successfully applied in the field of elasto-plastic behaviour of sheet for instance by Meuwissen et al. [13], Kajberg and Lindkvist [14], Haddadi et al. [15], Velay et al. [16], Dournaux et al. [17] and Lecompte et al. [18,19] on biaxial tests. Note that FEMU method using full-field measurements has been also introduced in other fields, e.g. for composite materials [20-22] or for structural dynamics [23,24].

The main purpose of the paper is to present and discuss a Finite Element Model Updated (FEMU) procedure for the identification of the elasto-plastic parameters of a 2024-T3 aluminium alloy. In the present purpose, an orthotropic Hill criteria [4] including an isotropic hardening is considered. On the one hand, standard tensile tests have provided a first set of parameters as a global response of the material. On the other hand, some of these parameters are optimised from the proposed FEMU procedure, as local responses of the material. These inverse identifications are based on different geometries from the literature which induce heterogeneous strain fields that can be measured thanks to Digital Image Correlation (DIC) technique. The aim of the work is to present in details the FEMU inverse identification procedure and to study the influence of the specimen shape providing various heterogeneous strain fields on the parameter identification of the isotropic hardening evolution. It is considered here only plane strain models and 2-D modified tensile test specimen geometries.

The outline of the paper is the following. First, the methodology is presented including the behaviour model and the full-field measurements method used. Next, the FEMU identification procedure is described. Last, results of the identification of the isotropic hardening parameters are presented and discussed depending on the specimen shapes. 


\section{ELASTOPLASTIC CONSTITUTIVE MODEL}

\subsection{Behaviour Model}

In the present paper, an elasto-plastic model in plane stress conditions is considered. It includes a Hill's yield criterion [4] and an isotropic hardening law defined as ${ }^{\dagger}$ :

$$
f=\sqrt{H\left(\sigma_{11}-\sigma_{22}\right)^{2}+F\left(\sigma_{22}\right)^{2}+G\left(\sigma_{11}\right)^{2}+2 N\left(\sigma_{12}\right)^{2}}-R
$$

where $\sigma_{i j}$ are the components of the Cauchy stress tensor, $\{F, G, H, N\}$ the Hill's coefficients and $R$ the isotropic hardening. $R$ is defined by the following Ludwig equation:

$$
\mathrm{R}=R_{0}+K\left(\varepsilon_{e q}^{P}\right)^{n}
$$

where $R_{0}$ is the yield stress, $K$ and $n$ are material parameters and $\varepsilon_{e q}^{P}$ is the equivalent plastic strain. This formulation is chosen because it allows to characterise the isotropic hardening evolution with only two parameters ( $K$ and $n$ ) independently of the yield stress.

\subsection{Identification from standard tensile tests}

The elastic parameters $(E, v)$, the isotropic hardening parameters $\left(K, n, R_{0}\right)$ and the plane anisotropy are first identified classically from standard tensile tests at three different angles $\alpha$ from the rolling direction $\left(0^{\circ}, 45^{\circ}, 90^{\circ}\right)$.

Tensile tests are carried out on an Instron 5800 servo-electric tensile testing machine with specimens machined by water jet cutter from a 2024-T3 aluminium sheet. Strains are measured by Digital Image Correlation (DIC) technique, described in detail in section 3.2. This technique allows to measure the longitudinal $\left(\varepsilon_{y y}\right)$ and transversal $\left(\varepsilon_{x x}\right)$ strains along with the time. About 130 images are acquired for each test (area of interest $12 \times 30 \mathrm{~mm}^{2}$, magnification factors 26 pixels/mm). DIC parameters are (see section 3.2): subset size 31 pixels, step size 10 pixels, and gauge length $(\mathrm{N}=5$ neighbouring matched points used for the strain computation, see section 3.2) $10 *(5-1)=40$ pixels $(1.5 \mathrm{~mm})$. The global strain value is computed as the mean spatial

\footnotetext{
${ }^{\dagger}$ It has been reported that the plastic anisotropy of a 2024 aluminium thin sheet was not well described by the yield functions of Hill48 potential [4], and other yield functions could be more suitable [5,6,26]. However the identification of their parameters is delicate and is not the subject of this study.
} 
strain value over all the calculated points in the area of interest. As standard tensile tests are assumed to provide homogeneous strain fields, the spatial standard deviation of the strain can characterise the uncertainty of the measurement. Note that it includes all the measurement errors coming from the shape of the specimen, the testing machine that can introduce bending, the DIC method, etc. Standard deviation of the strain was calculated, it increases with the strain magnitude and reaches a maximum value of about $0.2 \%$ for $10 \%$ of strain in the direction. The global strain values are used to calculate the anisotropy coefficients (or Lankford's coefficients) $r_{\alpha}$ and to determine the yield stresses $\sigma_{\alpha}$ in different directions. Lankford's coefficients $r_{\alpha}$ are obtained from the ratio between the plastic strain rate in the width (x) and in the thickness (z) directions, assuming an isochoric plasticity $\varepsilon_{z z}^{p}=1-\varepsilon_{x x}^{p}-\varepsilon_{y y}^{p}$.

Figure 1 presents the experimental tensile curves for the three angles $0^{\circ}, 45^{\circ}$ and $90^{\circ}$ from the rolling direction. These curves show a relatively weak anisotropic criterion and a hardening independent of the angles from the rolling direction.

[Fig. 1 about here.]

The yield stress values are taken as the conventional $0.2 \%$ offset yield stress values $\left(R_{p 0.2}\right)$. Then the identification of $K$ and $n$ is done from the overall three true stress / plastic strain curves, as presented in the sub-figure inserted in Fig. 1. The values of the identified parameters are reported in Table 1.

[Tab. 1 about here.]

Hill's coefficients are determined thanks to $r_{\alpha}$ and $\sigma_{\alpha}$ coefficients. The Hill48 criterion [4] can be expressed as

$$
f=\sigma_{\alpha} \sqrt{(H+G) \cos ^{4}(\alpha)+(F+H) \sin ^{4}(\alpha)+2(N-H) \cos ^{2}(\alpha) \sin ^{2}(\alpha)}-R_{0}-K\left(\varepsilon_{e q}^{p}\right)^{n}
$$

leading to the flow stress for $f=0$ 


$$
\sigma_{\alpha}=R_{0} / \sqrt{(H+G) \cos ^{4}(\alpha)+(F+H) \sin ^{4}(\alpha)+2(N-H) \cos ^{2}(\alpha) \sin ^{2}(\alpha)}
$$

The expression of the Lankford's coefficients is deduced from the flow rule $\varepsilon_{i j}{ }_{i j}=\lambda \frac{\partial f}{\partial \sigma_{i j}}$ according to the normality assumption, with $\varepsilon_{i j}^{\cdot p}$ are the components of the plastic strain tensor and $\lambda$ the plastic multiplier. It is given by

$$
r_{\alpha}=\frac{\varepsilon_{22}^{\cdot p}}{\varepsilon_{33}^{\cdot p}}=\frac{(G+4 H+F-2 N) \sin ^{2}(\alpha) \cos ^{2}(\alpha)-H}{-(G \cos (\alpha)+F \sin (\alpha))}
$$

The set of Hill's parameters $F, G, H$ and $N$ are identified using Eq. (2.4) and (2.5). Four independent equations are needed. In this study as proposed in [6] we use the three measured values of the yield stresses $\sigma_{0}, \sigma_{45}$ and $\sigma_{90}$ given in Table 1 with respect to Eq. (2.4) and the value of $r_{45}=0.904$ with respect to Eq. (2.5). The set of identified Hill's parameters are presented in Table 2.

[Tab. 2 about here.]

\section{IDENTIFICATION FROM FULL-FIELD MEASUREMENTS}

\subsection{Methodology}

The procedure describes hereafter leads to obtain the optimised values of the behaviour parameters $P$ in the case which the strain field resulting from the mechanical test is downright heterogeneous. It is based on the comparison between the FEM numerical solutions and the measurement results of both the nodal displacements and the global force. The procedure is resumed in Fig. 2.

[Fig. 2 about here.] 
The specimen geometry is first defined by measuring its 3-D shape in the concerned area (called area of interest, AOI) with the 3-D DIC method (or stereo-correlation method, see section 3.2). It is verified that the out-of-plane displacements are always negligible so that the hypothesis of plane stress conditions is relevant. The FEM meshing (nodes $N_{k}$ ) is then built from this 2-D contour (see section 3.3). The first calculation is performed with an initial set of constitutive parameters $P_{0}$. They could be provided by simple tensile tests as previously presented (see section 2), but not necessarily. Imposed boundary conditions are directly set to the measured node displacements. After the calculation, the $N_{k}$ nodal displacements $\overrightarrow{U_{k}}=\left(U_{1} \vec{x}+U_{2} \vec{y}\right)$ of the AOI are collected and compared to their experimental counterparts. A specific procedure allows to catch the correct DIC grid nodes corresponding to the $N_{k}$ FEM mesh nodes (see section 3.3). The difference is then quantified with a cost function $\phi(P)$ which is the sum of the squared differences between numerical and experimental data (nodal displacements inside the AOI and global force). Moreover, it is considered in this work that an identification test can be done from many tensile steps (or "deformation maps") $N_{i}$ : at each step $i$ corresponds a state of deformation under a tensile loading (typically, an imposed displacement step of the crosshead of the testing machine), leading to the measurement of the displacement field by 3-D DIC (by recording a stereo pair of images) and the total tensile force by the force sensor of the machine.

Thus the overall cost function is defined with a uniform repartition of weighting between displacements and force, as proposed for example in [14,15]:

$$
\phi(P)=\left[\phi_{F}(P)+\phi_{U}(P)\right] / 2
$$

The first term is the mean, over each considered steps $i$, of the normalised Euclidian distance between the calculated (superscript $\operatorname{sim}$ ) and measured (superscript exp) total force $F$ :

$$
\phi_{F}(P)=\frac{1}{N_{i}} \sum_{i=1}^{N_{i}}\left\|\frac{F^{\text {exp }}-F^{\text {sim }}(P)}{F^{\exp }}\right\|_{i}
$$


The second term is the mean, over each considered steps $i$, of the normalised Euclidian distance between the calculated (sim) and measured (exp) displacement $\overrightarrow{U_{k}}=\left(U_{1} \vec{x}+U_{2} \vec{y}\right)_{k}$ of the $N_{k}$ nodes:

$$
\phi_{U}(P)=\frac{\sqrt{\frac{1}{N_{i} N_{k}} \sum_{k, i=1}^{N_{k}, N_{i}}\left(\left(U_{1}^{s i m}(P)-U_{1}^{\exp }\right)_{i, k}^{2}+\left(U_{2}^{\sin }(P)-U_{2}^{\exp }\right)_{i, k}^{2}\right)}}{\sqrt{\frac{1}{N_{i} N_{k}} \sum_{k, i=1}^{N_{k}, N_{i}}\left(U_{1}^{\exp }{ }^{2}+U_{2}^{\exp }\right)_{i, k}-\left(\frac{1}{N_{i} N_{k}} \sum_{k, i=1}^{N_{k}, N_{i}} \sqrt{\left(U_{1}^{\exp }{ }^{2}+U_{2}^{\exp }\right)_{i, k}}\right)^{2}}}
$$

Two important remarks can be done about the cost function. First, the displacement cost function $\phi_{U}(P)$ is not normalised as usual by the experimental displacement $U_{i, k}^{\text {exp }}$ as proposed e.g. in [17] or by the experimental maximum displacement [21]. It is normalised by the standard deviation of the experimental displacements in the AOI, because as large rigid body motion can be observed for the considered AOI of the specimen which can be a reduced part of the specimen, the displacement cost function is underestimated with respect to $\phi_{F}(P)$. Note that Kajberg and Lindkvist [14] have proposed a displacement cost function normalised by $\left|U_{i}^{\text {exp } \max }\right|-\left|U_{i}^{\text {exp, mean }}\right|$ that also takes into account rigid body motions. $\phi_{U}(P)$ and $\phi_{F}(P)$ have thus the same weight. Second, in the literature, instead of displacement fields, strain fields are often considered directly in the cost function [15,18-20]. In such a case, it is thus implicitly assumed that (i) the formulations to calculate strains and (ii) the strain spatial resolution (or gauge length) are the same for both the DIC algorithm and the FEM post-processor. These two points are generally impossible to ensure because softwares are different. Furthermore, it is known that signal-to-noise ratio of measured displacement fields is larger than the one of the strain fields. Thus the introduction of the displacement field in the cost function avoids to add further errors in the identification procedure.

The overall cost function is then minimised iteratively with respect to the behaviour parameter set $P$. In this work, a zero-order optimisation method (Nelder-Mead simplex method) is used instead of a first-order gradient method. As there is no analytical solution for the cost function, whatever the gradient method the Jacobean or Hessian matrixes would be 
evaluated by finite differences schemes, which would add numerical errors. By using a direct optimisation method, numerical errors are minimised to the possible detriment of an increase in the computation time. The convergence criterion (difference between overall cost function values of two successive iterations) is set to $10^{-3}$.

\subsection{Digital Image Correlation}

In this work, 3-D Digital Image Correlation (3-D DIC) is used for the displacement field measurements. It should be noted that 3-D DIC is used instead of standard 2-D DIC for planar specimen, for three main reasons: (a) in 2-D DIC, the specimen must be positioned parallel to the camera sensor and must undergo a planar deformation without any out-of-plane displacement, which is difficult to guarantee in practice particularly when large displacement are expected (an apparent strain could be added to the real strain undergone by the specimen), (b) due to the calibration requirements of the stereo rig, lens distortions are also corrected for better accuracy measurements and (c) the 3-D DIC gives directly the shape of the specimen and the displacement fields in metric unit because the magnification factor is known after calibration. The (planar) shape of the specimen is used to define the right geometry and the AOI to be considered for the FEM. During the experiments, as previously discussed, a negligible out-of-plane displacement has been measured, so only the in-plane displacement is taking into account in this work.

The 3-D DIC method is based on both digital image correlation and stereovision [26]. This technique uses a DIC algorithm to determine point correspondences between two images of a specimen acquired from two rigidly bounded cameras. The correlation score is computed by measuring the similarity of a fixed subset window in the first image to a shifting subset window in the second one. A first-order two-dimensional shape function in the subset and a zero normalised sum of square difference (ZNSSD) correlation criterion are used. Sub-pixel correlation is performed using 6-tap spline grey level interpolation. After determining the calibration parameters for each camera as well as the 3-D relative position/orientation of the two cameras (pinhole model and radial distortion of 2nd order), the 3-D specimen shape can be reconstructed from the point correspondences using triangulation. To determine the 3-D 
displacement field, DIC is also used to determine point correspondences between the stereo pairs acquired before and after deformation. A complete description of the 3-D DIC technique can be found in the literature [26-28].

The stereo-rig is composed of two 8-bit digital AVT Dolphin F-145B cameras with a CCD resolution of $1,392 \times 1,040$ pixels. They are equipped with Tamron $28-200 \mathrm{~mm}$ f3.8-5.6 telephoto lens. The numerical image processing is carried out with the assistance of the Vic$3 \mathrm{D}^{\circledR}$ commercial software [29]. In order to capture identifiable images, a randomly distributed paint pattern is laid on the side of the specimen. For the measurements on geometries which induced heterogeneous strain fields, typical magnification factors are about 25 pixels $/ \mathrm{mm}$ and planar dimensions of the AOI are typically $24 \times 30 \mathrm{~mm}^{2}$. The square subset size which defines the displacement spatial resolution is chosen equal to 19 pixels, corresponding to about 0.76 $\mathrm{mm}$. The step size, which defines the pitch of the square grid of the measured points by DIC in the left image, is chosen equal to 5 pixels $(0.2 \mathrm{~mm})$. It is a compromise for obtaining a sufficiently dense displacement field without using an excessive amount of computer time. Eventually, strains can be computed as follow: at each matched point, an affine displacement function is fitted using a set of $N$ by $N$ neighbouring matched points chosen in a square surface. The Henky 2-D strain tensor is then computed using the estimated terms of the displacement function. These computations are done in a local coordinate system (aligned with a local tangent plane to the undeformed surface) obtained by a least-square plane fitting of this square surface. $N=5$ points (separated by a step of 5 pixels) is chosen because it is the smallest value permitted by the software, so the gauge length is equal to $5^{*}(5-1)=20$ pixels $(0.8 \mathrm{~mm})$.

A brief performance analysis of the measurement errors in the same configuration as the experiment (same camera positions, lighting, speckle, DIC parameters, etc.) was performed by analysing results of a no-motion experiment (errors from image noise) and a no-strain experiment (errors from a rigid body motion (RBM) of the specimen), as proposed in [30]. Results are summarized in Table 3 ( $U$ is the horizontal displacement, $V$ the vertical displacement). Note that a computation was also done to remove the RBM from the measured displacement field, giving a mean displacement equal to zero (see the third line in Table 3 ). 
Note also that this kind of analyses gives a lower bound of the standard displacement uncertainties. The maximum value that could be associated to the displacement standard uncertainty is $1.7 \mu \mathrm{m}$ (lower than 0.07 pixel), which is the standard deviation of the vertical displacement. This value is within the same range as reported in [30].

\subsection{Finite Element Model}

The FEM simulations are performed with ABAQUS ${ }^{\mathrm{TM}}$ software. An elasto-plastic model is considered corresponding to the constitutive equations (Eq. (2.1) and (2.2)) previously presented. Quadrangular shell elements are considered. The FEM meshing (nodes $N_{k}$ ) is built from the 2-D contour of the (quasi) planar shape of the specimen measured by 3-D DIC, so the investigation area meshed for the model corresponds to the AOI determined by the 3-D DIC measurement. To calculate the cost function and to impose the displacement boundary conditions, the knowledge of the $x$ and $y$ components of the measured displacement by 3-D DIC is needed at each node of the FEM meshing. As the DIC experimental grid (step $0.2 \mathrm{~mm}$ ) is obviously more refined than the FEM meshing, a bicubic interpolation of the measured displacement field is performed, giving for each FEM mesh node a precise evaluation of the experimental displacement vector. Finally, as previously presented, the displacement boundary conditions applied to the top and the bottom nodes of the meshed AOI are provided by the DIC measurement values. It should be noted that, because the discrete measurement points provided by the 3-D DIC are very close together, the data is highly redundant and only a part of the experimental data are used in the FEMU procedure itself by the mean of interpolation onto the FE mesh. This approach may not be optimum e.g. in term of noise measurement sensitivity. Some interesting approaches were proposed in the literature to reduce the dimensional representation of such measurements, by using shape feature vectors decomposed on Zerkine polynomials $[23,24]$ or proper orthogonal decomposition [31].

\section{RESULTS}

In the following, our FEMU identification procedure is applied to some of the specimen geometries from the literature $[13,14]$. These geometries inducing heterogeneous strain fields 
were tested in order to investigate the influence of the sample shape and of the strain field induced on the identification results.

\subsection{Case of Meuwissen specimen geometry}

The FEMU identification is first based on the specimen geometry proposed by Meuwissen et al. in 1998 [13]. This geometry, presented in Fig. 3, was designed by the authors to induce heterogeneous strain fields in a tensile test configuration.

[Fig. 3 about here.]

The test is carried out on an Instron servo-electric tensile testing machine. The specimen is machining by water jet cutter on a 2024-T3 aluminium sheet with the rolling direction perpendicular to the length of the specimen. During the test, images are acquired and global force values are recorded. After processing of the pairs of images, the experimental planar displacement fields are deduced. As an illustration, Fig. 4(a) presents the vertical experimental displacement field obtained by Vic-3D ${ }^{\circledR}$ for the last tensile step, superimposed on the black and white speckle pattern. For the same solicitation, Fig. 4(b) presents the FEM simulated vertical displacement field based on the same AOI and the measured displacements as boundary conditions, allowing to take into account rigid body motions.

[Fig. 4 about here.]

In the following only the isotropic hardening parameters $K$ and $n$ have been considered as variable parameters, and the other material parameters needed for the FEM simulations are set to the values provided by the 1D identification (see section 2.2 and Table 1 and 2). In a first step, many direct FE simulations are done with different couples of $K$ and $n$ parameters. For each simulation, the cost function is computed using the experimental measurements. Hence, the cost function map can be plotted versus $K$ and $n$ which gives an easy-to-read representation of the sensitivity of the procedure to the hardening parameters. 
To describe the whole range of the elasto-plastic domain, $N_{i}=20$ displacement fields / force measurements are used both in the direct FEM simulations (for plotting the sensitivity map) and in the FEMU procedure itself. These twenty load steps are shown in the experimental tensile curve formulated in terms of force - longitudinal mean strain and presented in Fig. 5. The mean strain calculation is performed over all the AOI.

[Fig. 5 about here.]

Figure 6 presents the results for $K$ in the range [200;1500] MPa with a step of $100 \mathrm{MPa}$ and $n$ in the range $[0.16 ; 1.06]$ with a step of 0.1 . It is observed a large valley-like region of minima for parameter values included in [0.25; 0.45] for $n$ and in [400; 900] MPa for $K$.

[Fig. 6 about here.]

In a second step, the optimisation procedure is performed until convergence. In order to investigate the influence of the initial parameter set, the optimisation procedure is performed by using several initial parameter sets $\mathrm{Pa}, \mathrm{Pb}$ and $\mathrm{Pc}$ defined in Table 4.

[Tab. 4 about here.]

For one of them, the cost function evolution is analysed. Figure 7 presents the force, displacement and overall cost function versus the iteration number (see Eq. (3.1)-(3.3)). For the twenty-five first iterations their values decrease. Then for the following iterations the force cost function goes on decreasing whereas the displacement cost function increases, until the convergence criteria are satisfied (79 iterations).

[Fig. 7 about here.]

This observation can be explained by the fact that, one the one hand, for the FEM simulations both the sample shape and the boundary conditions are provided by the 
experimental DIC measurements (position and displacement). Thus simulated displacement fields used in the displacement cost function formulation are always consistent with their experimental counterparts. On the other hand, simulated reaction forces induced by the displacement fields have to accommodate at each iteration considering the behaviour model and the measured force used in the force cost function formulation. The optimised parameter values result from a compromise between both forces and displacement fields. The associated parameter evolution is illustrated in Fig. 8.

[Fig. 8 about here.]

Results for the three initial parameter sets $\mathrm{Pa}, \mathrm{Pb}$ and $\mathrm{Pc}$ are presented in Table 4 and Fig. 6. Whatever the initial parameter set considered, the optimisation results are very close, with the following obtained mean values: about $K=989 \mathrm{MPa}$ and $n=0.5$. The inverse procedure is thus not sensitive to the initial conditions, and the optimised parameter set is situated at the lower position in the valley of minima (see Fig. 6). The parameter values found will be discussed further.

The influence of the number of experimental load steps taken into account in the FEMU procedure is now investigated. In the present study the total strain range is kept constant, as for all the cases at least the first and the last load step are included in the procedure, as it can be seen in Fig. 5. More precisely, five combinations have been studied: 5, 10 (first and second set), 15 and 20 load steps. It has been verified that all the FEMU computations converge properly (for various initial parameter sets), even for the former combination of only 5 load steps. In Fig. 5 are described in detail the load steps corresponding to the five combinations, showing that the load steps of the first combination (5 load steps) are always included in the other combinations. Results of cost function values before and after optimisations as well as optimised parameters are presented in Table 5, only for the initial parameter set $\mathrm{Pb}$, as it has been shown previously that (in case of convergence) the choice of the initial parameter set presents a very small impact on the results. 
[Tab. 5 about here.]

On the contrary, the choice of the images / load steps influences in a relatively important way the results of the material parameter identification. For the parameter $K$ the mean value found is equal to about $981 \mathrm{MPa}$ with a standard deviation of $24 \mathrm{MPa}$, and a difference between max and min values upper than $64 \mathrm{MPa}$, namely $6.5 \%$ of the mean value. For the parameter $n$ the mean value found is equal to about 0.5 with no real significant variations. However these differences are not negligible and can be explained by the a priori choice of the load steps in the global tensile curve that can favour a region of the elasto-plastic curve. It is worth noting that this is not so trivial as at each step corresponds a heterogeneous strain field. Nevertheless, for instance, the first combination of the 10 load steps selected takes into account more steps in the region between the end of the elastic regime and the beginning of the plastic regime than the second combination (see Fig. 5), and the provided results are widely different. On the contrary, considering the 15 load steps combination, the five steps added to the second combination are steps located in the fully developed plasticity part, and the results found are very close. To summarize, it is likely that a lot of data (load steps) with an uniform distribution all over the loading range will allow to obtain a more realistic parameter set. Thus the final parameter set obtained for the 20 load steps combination ( $K=990 \mathrm{MPa}, n=$ $0.5)$ seems to be the more accurate. But there is clearly an important effect of the choice of the steps (experimental displacement fields and loads) as an input in the identification procedure, giving in our case more than $6.5 \%$ in discrepancy on one of the identified parameters.

Finally, it is observed in such a geometry under tensile loading that the large part of the experimental displacement field is provided by a global tensile (e.g. homogeneous) strain field with a relatively small level of strain, excepted near the edges of the notches. This is shown in Fig. 9 (a) which depicts the experimental longitudinal strain field for the last load step (DIC parameters are the same as presented in section 3.2: subset size 19 pixels, subset separation 5 pixels, and gauge length $5 *(5-1)=20$ pixels, magnification factor 24 pixels $/ \mathrm{mm})$ : a large part of the strain field is less than $2.6 \%$ while maximum values are close to $17 \%$. It is expected that better or more realistic results of the inverse parameter identification may be obtained if we 
consider mainly areas where the strain field is highly heterogeneous and the strain values are uniformly distributed all over the strain range. To test the sensitivity to the choice of the area of the displacement field used for the evaluation of the displacement cost function, a new limited area has been designed (see Fig. 9 (b)) and only the displacement field within this area has been used in the FEMU procedure. The same twenty load steps have been used.

[Fig. 9 about here.]

Whatever the initial parameter set considered, the optimisation results are as expected very close, with the following mean values: about $K=936 \mathrm{MPa}$ and $n=0.49$. We observe an almost unchanged value for the latter parameter but a decrease of more than $5 \%$ for the parameter $K$ which was $990 \mathrm{MPa}$. This discrepancy which depends on the choice of the area of the displacement field used in the FEMU procedure is of the same order of magnitude as the one relating to the choice of the load steps used in the FEMU procedure. Nevertheless, the final parameter set obtained for this specimen geometry ( $K=936 \mathrm{MPa}, n=0.49)$ seems to be the more accurate, because whatever the load step the area used in the FEMU procedure displays a more uniform and larger distribution of strain level. But it should be reminded that there is a relatively important effect of the choice of this area as an input in the identification procedure. Note also that the strain range provided by each load step is probably an important task and it will be discussed in the section 5 .

\subsection{Case of Haddadi specimen geometry}

The identification procedure is now applied on the geometry proposed more recently by Haddadi et al. [15,32]. As we have seen that the load step and the area of the displacement field impact the parameter identification result, it is also supposed that the geometry plays a crucial role on it. The geometry of the specimen is presented in Fig. 10.

[Fig. 10 about here.] 
The test is also carried out on the Instron servo-electric tensile testing machine with a water jet cutter 2024-T3 specimen. Figure 11(a) presents the AOI and the major strain field obtained by Vic-3D ${ }^{\circledR}$ for the last tensile step, superimposed on the black and white speckle pattern (DIC parameters: subset size 19 pixels, subset separation 5 pixels, and gauge length 20 pixels, magnification factor 26 pixels $/ \mathrm{mm}$ ). Figure 11(b) presents the FEM mesh. Ten displacement fields / load steps have been extracted from the experiment and used both in the direct FEM simulations (900 nodes) and in the FEMU procedure itself. For this geometry too, the displacement field used in the displacement cost function is restricted to the sub-area illustrated in Fig. 11 where significant strain level occurs.

[Fig. 11 about here.]

For this experiment, the sensitivity map has also been calculated in the same ranges for the $(K, n)$ parameters, as shown in Fig. 12.

[Fig. 12 about here.]

This sensitivity map still presents a valley-like region of minima for parameter values included in $[0.3 ; 0.6]$ for $n$ and in $[500 ; 1500] \mathrm{MPa}$ for $K$. The optimisation procedure is then performed until convergence, by using the same initial three parameter sets $\mathrm{Pa}, \mathrm{Pb}$ and $\mathrm{Pc}$ as before. Results are presented in Table 6. All initial parameter sets converge to the same couple: about $K=636 \mathrm{MPa}$ and $n=0.37$, for a cost function value close to $3.1 \times 10^{-2}$.

[Tab. 6 about here.]

The procedure is, as for the Meuwissen experiment, not sensitive to the initial conditions, and the optimised parameter set is situated at the lower position in the valley of minima. 
It should be noted that until this point, noiseless data were assumed. This case is not representative of actual data of kinematic measurement by DIC, and therefore it is relevant to analyse the effect of noise level on the identification results. This sensitivity study is done by directly adding a Gaussian noise to both measured displacement components. In fact, it has been observed in our DIC measurement results (e.g. rigid body motion experiments) an almost Gaussian distribution of the displacement field. In section 3.2, the displacement standard uncertainty was estimated to $1.7 \mu \mathrm{m}(0.066 \mathrm{pixel})$. So three representative noise magnitudes of the actual measurement uncertainties are chosen: $0.033,0.066$ and 0.1 pixel, respectively corresponding to $0.86,1.7$ and $2.6 \mu \mathrm{m}$ knowing the magnification factor. Results of the FEMU procedure using noisy full-field displacement fields are inserted in Fig. 12. A single random draw for the noisy displacement field is done for noise levels corresponding to standard deviations of 0.86 and $1.7 \mu \mathrm{m}$. Eight random draws are performed for the larger noise level (standard deviation of $2.6 \mu \mathrm{m}$ ). As illustrated in Fig. 12, optimised parameter sets depend both on the noise magnitude and on the random draw. All the results appear to be on a line inside the valley of minima. For the smaller noise level the result is very close to the result without noise, then the parameter values decrease as the noise level increases. These simulations can also give an idea of parameter uncertainties from the results of the eight random draws for the larger noise magnitude. For the parameter $K$ the mean value found is equal to $611 \mathrm{MPa}$ with a difference between max and min values upper than $25 \mathrm{MPa}$, namely $4 \%$ of the mean value. For the parameter $n$ the mean value found is equal to about 0.35 with a difference between max and min values equal to 0.013 , also about $4 \%$ of the mean value. $K$ decreases from 636 to $611 \mathrm{MPa}$ (4\%) and $n$ from 0.37 to $0.35(5 \%)$. To summarise, adding an uncertainty in the DIC displacement fields of 0.1 pixel decreases both parameters of about 4 percent (bias) with a parameter uncertainty also about 4 percent. Note that the noisy displacement fields modify not only the displacement objective function but also the force objective function because boundary conditions of the FE model are also impacted. The parameter values found will be discussed in the next section.

\section{DISCUSSION}


In this study, the identification methodology was conducted on various geometries. Both of them consider a global uniaxial loading but with a heterogeneous strain field due to the particular shape of the specimen (e.g. Meuwissen and Haddadi specimens).

Focusing on results presented in Fig. 6 and Table 4 related to the Meuwissen experimental mechanical test, four comments emerge. First, a unique optimised solution is found. It shows that the inverse procedure is not sensitive to the initial conditions, even if a large valley-like area of minima is observed. Moreover, it is shown (see Table 5) that the choice of the steps (experimental displacement fields and loads) influences the identification procedure results, leading to more than $6.5 \%$ in discrepancy on the parameter $K$. The third important point is relative to the area of the displacement field which should be used for the evaluation of the displacement cost function. We show that it induces a decrease of the parameter $K$ of more that $5 \%$ ( $K$ varies from $990 \mathrm{MPa}$ to $936 \mathrm{MPa}$ ). Finally, the optimised parameter set $(K=936 \mathrm{MPa}$ and $n=0.49)$ is quite different to the one $(K=769 \mathrm{MPa}$ and $n=0.62)$ identified from the simple tensile tests.

Focusing now on results presented in Fig. 12 and Table 6 related to the Haddadi experimental mechanical test, three comments can be done. First, a unique optimised solution is also found. It shows that the inverse procedure is not anymore sensitive to the initial conditions, even if a large valley-like area of minima is also observed. Secondly, it is noticed (see the plot inserted in Fig. 12) that the inverse method is noise sensitive. By adding a noise of 0.1 pixel in the input displacement fields, it is shown that systematic and random errors associated to the parameters $K$ and $n$ are about $4 \%$ of their magnitudes. Finally, the optimised parameter set $(K=636 \mathrm{MPa}$ and $n=0.37)$ is quite different to the one identified from the simple tensile tests.

To summarise, the FEMU procedure is obviously sensitive to the experimental input data: (i) number of load steps, (ii) area of interest to be considered for computing the displacement cost function and (iii) noise related to the measured displacement fields. All these factors impairs the optimised parameter results, but the dispersion of the results remain all the same 
acceptable: each factor has an effect of more or less $5 \%$ of the magnitude of the parameters. We are going to show that the effect of the specimen geometry is much more important.

The influence of the specimen geometry is analysed by comparing results of the identifications based on the Meuwissen and the Haddadi geometries (see Fig. 6 and 12 and Table 4 and 6). Sensitivity maps present both a valley of minima but not exactly in the same zone. For the Haddadi geometry, the optimised parameter set $(K=636 \mathrm{MPa}$ and $n=0.37)$ is clearly different to the one identified from the Meuwissen geometry $(K=936 \mathrm{MPa}$ and $n=$ 0.49 ) and to the one identified from a standard simple tensile test ( $K=769 \mathrm{MPa}$ and $n=0.62$ ). However, for both non-standard geometries, the strain heterogeneities and the strain levels are also clearly not the same. This is illustrated in Fig. 13 where histograms of the experimental major strain levels are plotted for both geometries. Plot of the major strain versus minor strain is also inserted in Fig. 13.

[Fig. 13 about here.]

In both cases, only strain values in the limited areas previously defined (areas of large strain heterogeneities used in the FEMU procedures) and (for simplicity) only five load steps regularly distributed from the first to the last load step are analysed. Both histograms can be compared because frequencies are normalized. As it can be seen in Fig. 15, both geometries present almost the same major strain range, from $0.3 \%$ to $16 \%$ for Meuwissen and $18 \%$ for Haddadi. It is also observed that the Meuwissen geometry exhibits mainly strains in the $0.3 \%$ $7 \%$ range because the large major strains induced by this geometry are very spatially localised near the notches: only a few physical points in the material are fully strained plastically. On the contrary, the Haddadi geometry presents a more uniform strain distribution in the whole $0.3 \%$ $18 \%$ range. This spatially distributed heterogeneity of the strain in the gauge area seems to have an important impact on the inverse identification. This leads to obtain from these two sample shapes different identified parameter sets. Concerning the strain paths diversity, it can be noted that both geometries exhibit in a same manner strains between uniaxial tensile and plane strain, as it can be seen in the plot of the major vs. minor strain inserted in Fig. 15. This 
zone described by all strain paths remains far from the plane strain conditions. Their strain levels, as previously discussed, are clearly different, confirming large differences between Meuwissen and Haddadi geometries. Furthermore, it is worth pointing out that a simple tensile test explores a unique strain path whereas a heterogeneous tensile test ensures a diversity of strain paths. As a consequence, identification results show a noticeable difference between the standard tensile test and the heterogeneous tensile tests.

To summarize, it is believed that both strain ranges and differences in the geometry of the specimens that induce heterogeneities of the strain fields and diversities of the strain paths explain the differences in the optimised hardening parameters. In such a context, the choice of relevant specimen geometries and tests will be an issue to investigate. The second issue that would be interesting to investigate is a systematic estimation of the uncertainty in the optimised parameters, that could be achieved by providing robust development of error propagation calculus or by using some Monte Carlo approaches.

\section{CONCLUSION}

The present study has shown that it is possible to identify isotropic hardening parameters from full-field displacement measurements for aluminium sheet 2024-T3. For that, an identification procedure based on FEM simulations and displacement fields measured by 3-D DIC is developed. The identification capability is studied through several specimen geometries from the literature. These geometries were selected because they generate heterogeneous strain fields and various strain paths under tensile loading. We have shown that (i) the choice of the steps (experimental displacement fields and loads) as an input in the FEMU procedure, (ii) the choice of the area of the displacement field used for the evaluation of the cost function and (iii) noise related to the measured displacement fields have an impact on the identified parameters of more or less $5 \%$. The influence of the specimen geometry seems to be more crucial and identified parameters are clearly dependent of it. We have shown that both strain ranges and differences in the specimen shape that induce heterogeneities of the strain fields and diversities of the strain paths can explain the differences in the optimised hardening parameters. In our 
opinion, the Haddadi geometry seems to be the more relevant because it exhibits a more uniform heterogeneous strain distribution in the whole strain range than the Meuwissen geometry for which high strain gradients are very spatially localised. The identification of material parameters using full-field measurements and total force may a priori provide a more accurate behaviour model and therefore may improve the prediction given by FEM simulation. But this identification technique will need a relevant choice of the identification strategy that includes the definition of relevant specimen geometries and tests. This will be the challenge for future works.

\section{ACKNOLEDGMENTS}

The authors wish to acknowledge Pr. Jean-José Orteu and Correlated Solutions Inc. for providing the Vic-3D ${ }^{\circledR}$ software. The authors are grateful to Pr. Gérard Bernhart, the "MP05 advance forming" project (EMC2 French Competitivity pole) and the French Ministry of Industry for their technical and financial support.

\section{REFERENCES}

1. Cloud, G. Optical Methods of Engineering Analysis. Cambridge University Press, 1998, ISBN 0-521-45087-X.

2. Rastogi, P. Photomechanics. Springer Verlag, 1999, ISBN 3-540-65990-0.

3. Grédiac, M. The use of full-field measurement methods in composite material characterization: interest and limitations. Composites Part A, 2004, 35, 751-761.

4. Hill, R. A theory of the yielding and plastic flow of anisotropic metals. Proc. Royal Soc. London. Series A, Math. Phys. Sci., 1948, 193(1033), 281-297.

5. Barlat, F., Lege, D.J. and Brem, J.C. A six-component yield function for anisotropic materials, Int. J. Plasticity, 1991, 7, 693-712. 
6. Leacock, A. G. A mathematical description of orthotropy in sheet metals. J. Mech. Phys. Solids, 2006, 54, 425-444.

7. Avril, S., Bonnet, M., Bretelle, A.-S., Grédiac, M., Hild, F., Ienny, P., Latourte, F., Lemosse, D., Pagano, S., Pagnacco, E. and Pierron, F. Overview of Identification Methods of Mechanical Parameters Based on Full-field Measurements, Exp. Mech., 2008, 48, 381-402.

8. Claire, D., Hild, F. and Roux, S. A finite element formulation to identify damage fields: the equilibrium gap method. Int. J. Num. Methods Eng., 2004, 61(2), 189-208.

9. Latourte, F., Chrysochoos, A., Pagano, S. and Wattrisse, B. Elastoplastic Behavior Identification for Heterogeneous Loadings and Materials. Exp. Mech., 2008, 48, 435449.

10. Grédiac, M. and Pierron, F. Applying the virtual field method to the identification of elasto-plastic constitutive parameters. Int. J. Plasticity, 2006, 22, 602-627.

11. Avril, S., Pierron, F., Pannier, Y. and Rotinat, R. Stress Reconstruction and Constitutive Parameter Identification in Plane-Stress Elasto-plastic Problems Using Surface Measurements of Deformation Fields, Exp. Mech., 2008, 48, 403-419.

12. Pierron, F. Identification of Poisson's ratios of standard and auxetic low-density polymeric foams from full-field measurements, J. Strain Analysis, 2010, 45(4), 233253.

13. Meuwissen, M.H.H., Oomens, C.W.J., Baaijens, F.P.T., Petterson, R. and Janssen, J.D. Determination of the elasto-plastic properties of aluminium using a mixed numericalexperimental method. J. Mat. Proc. Tech., 1998, 75(1), 204-211.

14. Kajberg, J. and Lindkvist, G. Characterisation of materials subjected to large strains by inverse modelling based on in-plane displacement fields. Int. J. Sol. Struct., 2004, 41, 3439-3459.

15. Haddadi, H., Belhabib, S., Gaspérini, M. and Vacher, P. Identification of the Parameters of Swift Law Using Strain Field Measurements. In Proc. $8^{\text {th }}$ European Mechanics of Materials Conference on Material and structural identification from fullfield Measurements, 2005, Cachan, France. 
16. Velay, V., Robert, L., Schmidt, F., Hmida, S. and Vallet, T. Behaviour model identification based on inverse modeling and using Optical Full-field Measurements (OFFM). Proc. 10th ESAFORM conference, 2007, Zaragoza, Spain.

17. Dournaux, J.L., Bouvier, S., Aouafi, A. and Vacher, P. Full-field measurement technique and its application to the analysis of materials behaviour under plane strain mode. Mat. Sci. Eng. A, 2009, 500, 47-62.

18. Lecompte, D., Smits, A., Sol, H., Vantomme, J. and Van Hemelrijck, D. Mixed numerical-experimental technique for orthotropic parameter identification using biaxial tensile tests on cruciform specimens. Int. J. Solids Struct., 2007, 44, 1643-1656.

19. Cooreman, S., Lecompte, D., Sol, H., Vantomme, J. and Debruyne, D. Identification of Mechanical Material Behavior Through Inverse Modeling and DIC. Exp. Mech., 2008, 48, 421-433.

20. Molimard J., Le Riche R., Vautrin A., Lee J.R. Identification of the four orthotropic plate stiffnesses using a single open-hole tensile test. Exp. Mech., 2005, 45, 404-411.

21. Sztefek, P. and Olsson, R. Tensile stiffness distribution in impacted composite laminates determined by an inverse method. Compos. part A Appl. Sci. Manuf., 2008, 39(8), 1282-1293.

22. Fedele, R., Raka, B., Hild, F. and Roux, S. Identification of adhesive properties in GLARE assemblies by digital image correlation. J. Mech. Phys. Solids, 2009, 57(7), 1003-1016.

23. Wang, W., Mottershead, J.E., Ihle, A., Siebert, T. and Schubach, H.R. Finite element model updating from full-field vibration measurement using digital image correlation. J. Sound Vibr., 2011, 330(8), 1599-1620.

24. Wang, W., Mottershead, J.E., Sebastian, C.M. and Patterson, E.A. Shape features and finite element model updating from full-field strain data. Int. J. Sol. Struc., 2011, 48(11-12), 1644-1657.

25. Bron, F. and Besson, J. A yield function for anisotropic materials. Application to aluminum alloys. Int. J. Plasticity, 2004, 20, 937-963. 
26. Luo, P., Chao, Y., Sutton, M. and Peters W. Accurate measurement of threedimensional deformations in deformable and rigid bodies using computer vision. Exp. Mech., 1993, 30(2), 123-132.

27. Orteu, J.-J. 3-D Computer Vision in Experimental Mechanics. Opt. Lasers Eng., 2009, 47(3-4), 282-291.

28. Sutton, M.A., Orteu, J.-J. and Schreier, H.W. Image Correlation for Shape, Motion and Deformation Measurements - Basic Concepts, Theory and Applications. Springer, 2009.

29. Vic-3D ${ }^{\circledR} \quad$ software, Correlated Solutions Incorporated (C.S.Inc.), http://www.correlatedsolutions.com/, 2011.

30. Robert, L., Nazaret, F., Orteu, J.-J. and Cutard, T. Use of 3-D Digital Image Correlation to characterize the mechanical behavior of a Fiber Reinforced Refractory Castable. Exp. Mech., 2007, 47(6), 761-773.

31. Gogu, C., Haftka, R. T., Le Riche, R., Molimard, J., Vautrin, A. Dimensionality reduction of full fields by the principal components analysis, In Proc. $17^{\text {th }}$ International Conference on Composite Materials (ICCM17), Edinburgh, UK, 2009.

32. Belhabib, S., Haddadi, H., Gaspérini, M. and Vacher, P. Heterogeneous tensile test on elastoplastic metallic sheets: Comparison between FEM simulations and full-field strain measurements. Int. J. Mech. Sci., 2008, 50, 14-21. 


\section{Tables}

Table 1 Values of elastic properties, yield stresses and isotropic hardening parameters from standard tensile tests.

\begin{tabular}{|c|c|c|c|}
\hline Elasticity & \multicolumn{2}{|c|}{$E=71 \mathrm{GPa}$} & $=0.33$ \\
\hline Yield stresses $\left(R_{p 0.2}\right)$ & $\sigma_{0}=325 \mathrm{MPa}$ & $\sigma_{45}=297 \mathrm{MPa}$ & $\sigma_{90}=296 \mathrm{MPa}$ \\
\hline Isotropic hardening & \multicolumn{2}{|c|}{$K=769 \mathrm{MPa}$} & $n=0,62$ \\
\hline
\end{tabular}

Table 2 Values of Hill's coefficients.

\begin{tabular}{|c|c|c|c|}
\hline$F$ & $G$ & $H$ & $N$ \\
\hline 0.728 & 0.528 & 0.472 & 1.769 \\
\hline
\end{tabular}

Table 3 Measurement errors estimated from no-motion and no-strain (rigid body motion (RBM) of the specimen) experiments.

\begin{tabular}{|c|c|c|c|c|}
\hline & Mean $U$ & Standard dev. $U$ & Mean $V$ & Standard dev. $V$ \\
\hline No motion (noise) & $0.38 \mu \mathrm{m}$ & $0.44 \mu \mathrm{m}$ & $-0.66 \mu \mathrm{m}$ & $0.44 \mu \mathrm{m}$ \\
\hline Unstrained specimen & $7.2 \mu \mathrm{m}$ & $1.5 \mu \mathrm{m}$ & $13.4 \mu \mathrm{m}$ & $1.7 \mu \mathrm{m}$ \\
\hline
\end{tabular}




\begin{tabular}{|c|c|c|c|c|}
\hline (RBM of the specimen) & & & \\
\hline $\begin{array}{c}\text { Unstrained specimen with } \\
\text { removing RBM }\end{array}$ & $0 \mu \mathrm{m}$ & $0.7 \mu \mathrm{m}$ & $0 \mu \mathrm{m}$ & $1.6 \mu \mathrm{m}$ \\
\hline
\end{tabular}

Table 4 Results of the FEMU identification for three initial parameter sets (Meuwissen geometry, 20 experimental load steps considered in the identification procedure).

\begin{tabular}{|c|c|c|c|c|}
\hline & \multicolumn{2}{|c|}{ Initial } & \multicolumn{2}{|c|}{ Optimised } \\
\hline & Parameters & $\begin{array}{l}\text { Cost function } \\
\text { values }\end{array}$ & Parameters & $\begin{array}{c}\text { Cost function } \\
\text { values }\end{array}$ \\
\hline \multirow{2}{*}{$\mathrm{Pa}$} & $K=800 \mathrm{MPa}$ & \multirow{2}{*}{$6.949 \mathrm{E}-02$} & $K=988.4 \mathrm{MPa}$ & \multirow{2}{*}{$1.515 \mathrm{E}-02$} \\
\hline & $n=0.66$ & & $n=0.4986$ & \\
\hline \multirow{2}{*}{$\mathrm{Pb}$} & $K=200 \mathrm{MPa}$ & \multirow{2}{*}{ 4.944E-02 } & $K=989.6 \mathrm{MPa}$ & \multirow{2}{*}{$1.515 \mathrm{E}-02$} \\
\hline & $n=0.2$ & & $n=0.4989$ & \\
\hline \multirow{2}{*}{$\mathrm{Pc}$} & $K=1500 \mathrm{MPa}$ & \multirow{2}{*}{$9.438 \mathrm{E}-02$} & $K=989.6 \mathrm{MPa}$ & \multirow{2}{*}{$1.515 \mathrm{E}-02$} \\
\hline & $n=1$ & & $n=0.4989$ & \\
\hline
\end{tabular}

Table 5 Results of the FEMU identification for various experimental load steps considered in the identification procedure (Meuwissen geometry, initial parameter set $\mathrm{Pb}$ ). 


\begin{tabular}{|c|c|c|c|}
\hline Combinations & $\begin{array}{c}\text { Initial cost } \\
\text { function values }\end{array}$ & $\begin{array}{l}\text { Optimised } \\
\text { parameters }\end{array}$ & $\begin{array}{l}\text { Optimised Cost } \\
\text { function values }\end{array}$ \\
\hline \multirow{2}{*}{5 load steps } & \multirow{2}{*}{$5.116 \mathrm{E}-02$} & $K=953.8 \mathrm{MPa}$ & \multirow{2}{*}{$1.345 \mathrm{E}-02$} \\
\hline & & $n=0.4931$ & \\
\hline \multirow{2}{*}{$\begin{array}{c}10 \text { load steps }\left(1^{\text {st }}\right. \\
\text { combination })\end{array}$} & \multirow{2}{*}{$3.859 \mathrm{E}-02$} & $K=1017.9 \mathrm{MPa}$ & \multirow{2}{*}{$1.259 \mathrm{E}-02$} \\
\hline & & $n=0.5111$ & \\
\hline \multirow{2}{*}{$\begin{array}{c}10 \text { load steps }\left(2^{\text {nd }}\right. \\
\text { combination })\end{array}$} & \multirow{2}{*}{$5.051 \mathrm{E}-02$} & $K=966.6 \mathrm{MPa}$ & \multirow{2}{*}{$1.499 \mathrm{E}-02$} \\
\hline & & $n=0.4939$ & \\
\hline \multirow{2}{*}{15 load steps } & \multirow{2}{*}{$5.698 \mathrm{E}-02$} & $K=976.6 \mathrm{MPa}$ & \multirow{2}{*}{$1.556 \mathrm{E}-02$} \\
\hline & & $n=0.4956$ & \\
\hline \multirow{2}{*}{20 load steps } & \multirow{2}{*}{ 4.944E-02 } & $K=989.6 \mathrm{MPa}$ & \multirow{2}{*}{$1.515 \mathrm{E}-02$} \\
\hline & & $n=0.4989$ & \\
\hline
\end{tabular}

Table 6 Results of the FEMU identification for three initial parameter sets (Haddadi geometry, 10 experimental load steps considered in the identification procedure).

\begin{tabular}{|c|c|c|c|c|}
\hline \multirow{2}{*}{} & \multicolumn{2}{|c|}{ Initial } & \multicolumn{2}{c|}{ Optimised } \\
\cline { 2 - 4 } & Parameters & $\begin{array}{c}\text { Cost function } \\
\text { values }\end{array}$ & Parameters & $\begin{array}{c}\text { Cost function } \\
\text { values }\end{array}$ \\
\hline \multirow{2}{*}{$\mathrm{Pa}$} & $K=800 \mathrm{MPa}$ & \multirow{2}{*}{$1.123 \mathrm{E}-01$} & $K=635 \mathrm{MPa}$ & \multirow{2}{*}{$3.087 \mathrm{E}-02$} \\
\cline { 2 - 2 } & $n=0.66$ & & $n=0.370$ & \\
\hline \multirow{2}{*}{$\mathrm{Pb}$} & $K=200 \mathrm{MPa}$ & \multirow{2}{*}{$1.8599 \mathrm{E}-01$} & $K=637 \mathrm{MPa}$ & \multirow{2}{*}{$3.087 \mathrm{E}-02$} \\
\cline { 2 - 2 } & $n=0.2$ & & $n=0.371$ & \multirow{2}{*}{$3.087 \mathrm{E}-02$} \\
\hline \multirow{2}{*}{$\mathrm{Pc}$} & $K=1500 \mathrm{MPa}$ & \multirow{2}{*}{$1.4426 \mathrm{E}-01$} & $K=636 \mathrm{MPa}$ & \\
\cline { 2 - 2 } & \multicolumn{2}{|c|}{$n=1$} & $n=0.370$ & \\
\hline
\end{tabular}




\section{Table caption}

Table 1 Values of elastic properties, yield stresses and isotropic hardening parameters from standard tensile tests.

Table 2 Values of Hill's coefficients.

Table 3 Measurement errors estimated from no-motion and no-strain (rigid body motion (RBM) of the specimen) experiments.

Table 4 Results of the FEMU identification for three initial parameter sets (Meuwissen geometry, 20 experimental load steps considered in the identification procedure).

Table 5 Results of the FEMU identification for various experimental load steps considered in the identification procedure (Meuwissen geometry, initial parameter set $\mathrm{Pb}$ ).

Table 6 Results of the FEMU identification for three initial parameter sets (Haddadi geometry, 10 experimental load steps considered in the identification procedure). 


\section{Figure captions}

Fig. 1 Experimental tensile curves for three angles $\left(0^{\circ}, 45^{\circ}\right.$ and $\left.90^{\circ}\right)$ from the rolling direction.

The sub-figure (true stress vs. plastic strain) shows the experimental dots measured by DIC and the fitted Ludwig isotropic hardening in solid lines.

Fig. 2 Flow-chart of the Finite Element Method Updated (FEMU) identification procedure.

Fig. 3 Geometry of the Meuwissen specimen.

Fig. 4 Meuwissen specimen vertical displacement fields for the last experimental load step. (a)

Measured by 3-D DIC. (b) FEM simulation result taking into account the measured displacement as boundary conditions.

Fig. 5 Experimental tensile curve (force - mean longitudinal strain) obtained for the

Meuwissen specimen in solid line. The number of load steps considered in the identification procedure are superimposed in the curve (markers).

Fig. 6 Sensitivity map calculated using direct FEM simulations and experimental displacement fields (20 experimental load steps) for the Meuwissen specimen geometry: overall cost function evolution versus $K$ and $n$. Results of the FEMU identification for the three initial parameter sets $\mathrm{Pa}, \mathrm{Pb}$ and $\mathrm{Pc}$ are superimposed in the figure (20 experimental load steps included in the identification procedure, see Fig. 5).

Fig. 7 Evolution of the force, displacement and overall cost functions during the FEMU optimisation process (initial parameter set $\mathrm{Pb}$ ).

Fig. 8 Evolution of the parameters $K$ and $n$ during the FEMU optimisation process (initial parameter set $\mathrm{Pb}$ ).

Fig. 9 Meuwissen geometry. (a) Strain field (major strain) measured by DIC for the last experimental load step. The sub-area (white frame) corresponds to the area of the displacement field used for the evaluation of the displacement cost function. (b) FEM mesh. The filled points correspond to the FEM nodes used for the evaluation of the displacement cost function.

Fig. 10 Geometry of the Haddadi specimen.

Fig. 11 Haddadi geometry. (a) Strain field (major strain) measured by DIC for the last experimental load step. The sub-area (white frame) corresponds to the area of the 
displacement field used for the evaluation of the displacement cost function. (b) FEM mesh. The filled points correspond to the FEM nodes used for the evaluation of the displacement cost function.

Fig. 12 Sensitivity map calculated using direct FEM simulations and experimental displacement fields (ten experimental load steps) for the Haddadi specimen geometry: overall cost function evolution versus $K$ and $n$. Results of the FEMU identification for the three initial parameter sets $\mathrm{Pa}, \mathrm{Pb}$ and $\mathrm{Pc}$ are superimposed in the figure (ten experimental load steps included in the identification procedure). A zoom showing the effect of noisy kinematic fields is inserted.

Fig. 13 Histogram of the experimental major strain levels measured by DIC for both Meuwissen and Haddadi geometries. Inserted is the plot of the experimental major strain versus minor strain. Each strain fields investigated corresponds to the sum of five load steps regularly distributed between the first and the last load step. 


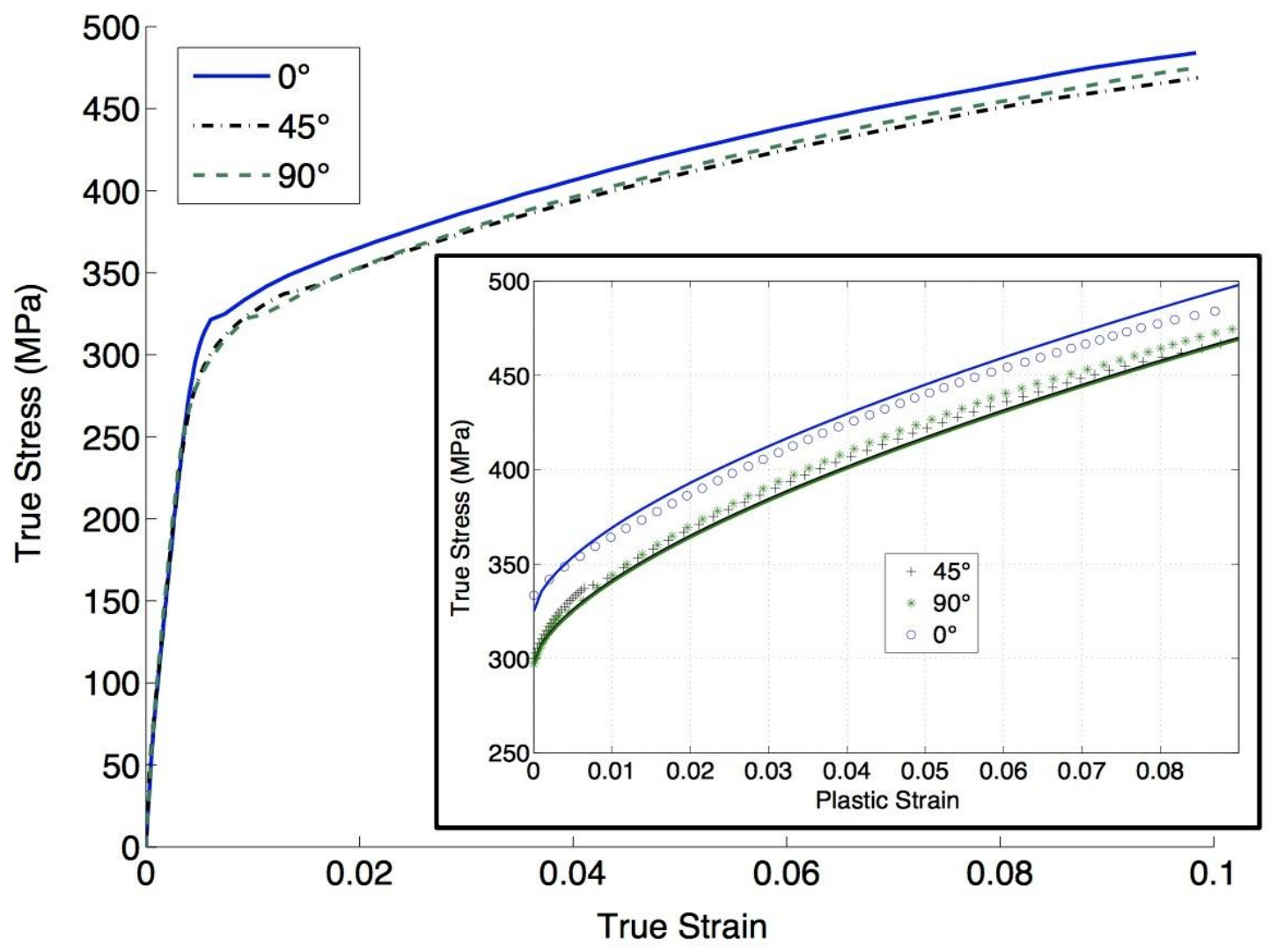

Figure 1. Experimental tensile curves for three angles $\left(0^{\circ}, 45^{\circ}\right.$ and $\left.90^{\circ}\right)$ from the rolling direction. The sub-figure (true stress vs. plastic strain) shows the experimental dots measured by DIC and the fitted Ludwig isotropic hardening in solid lines. 


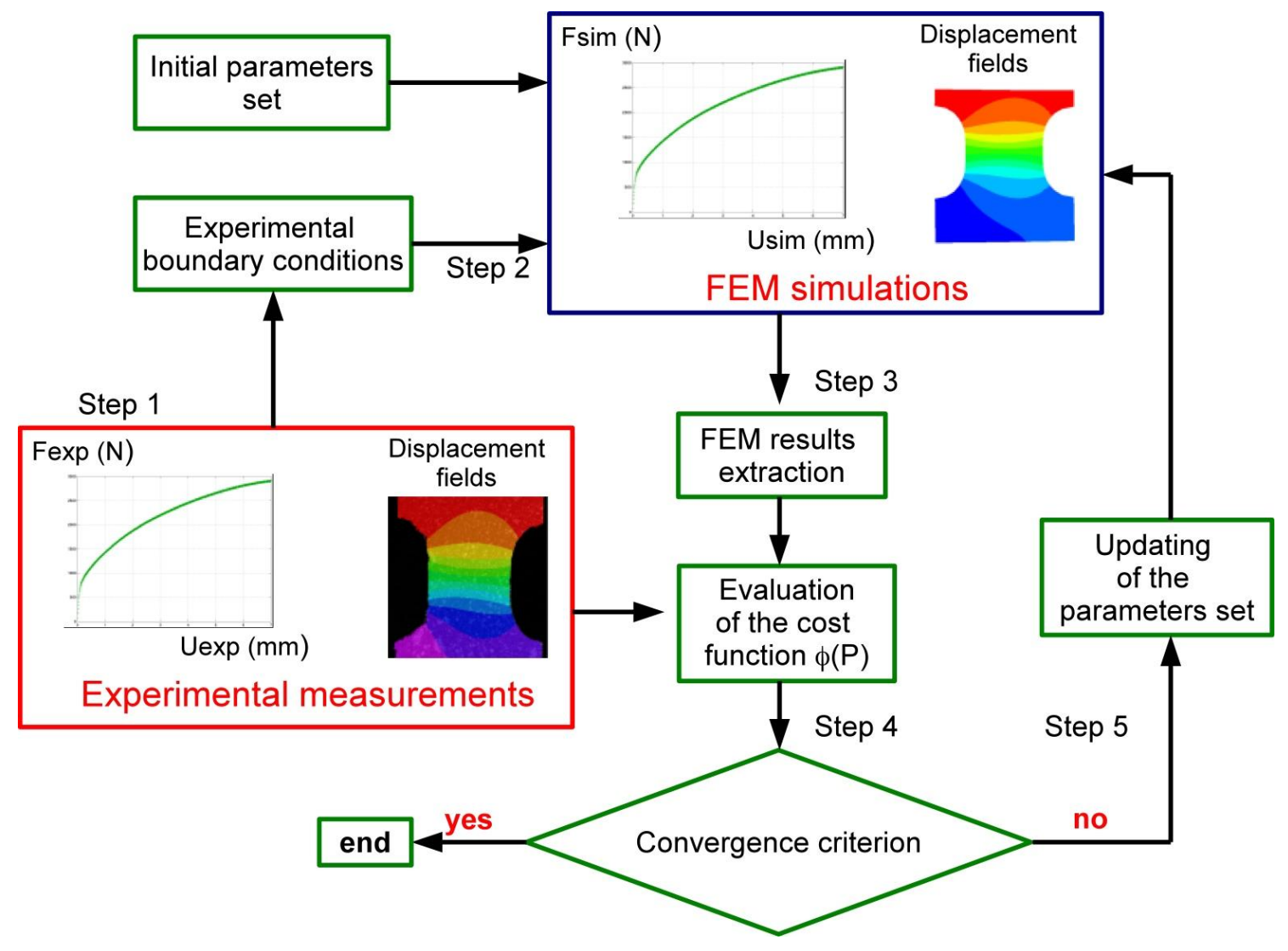

Figure 2. Flow-chart of the Finite Element Method Updated (FEMU) identification procedure. 


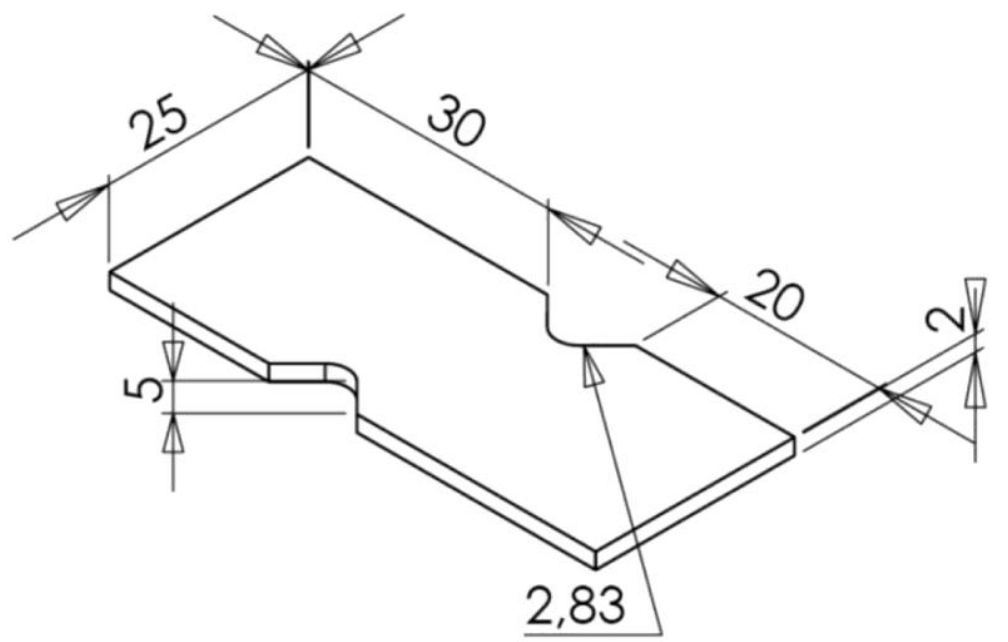

Figure 3. Geometry of the Meuwissen specimen. 

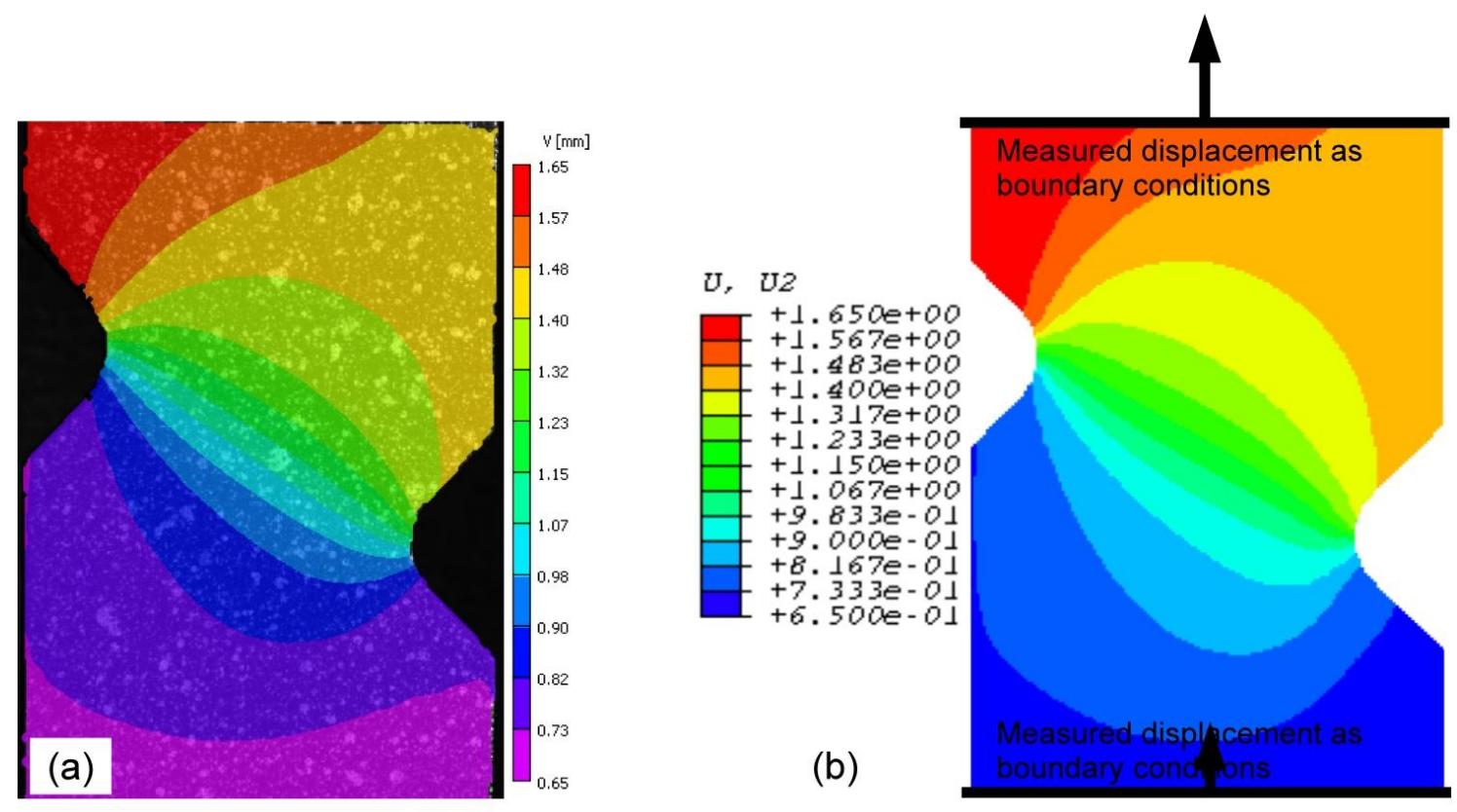

Figure 4. Meuwissen specimen vertical displacement fields for the last experimental load step. (a) Measured by 3-D DIC. (b) FEM simulation result taking into account the measured displacement as boundary conditions. 


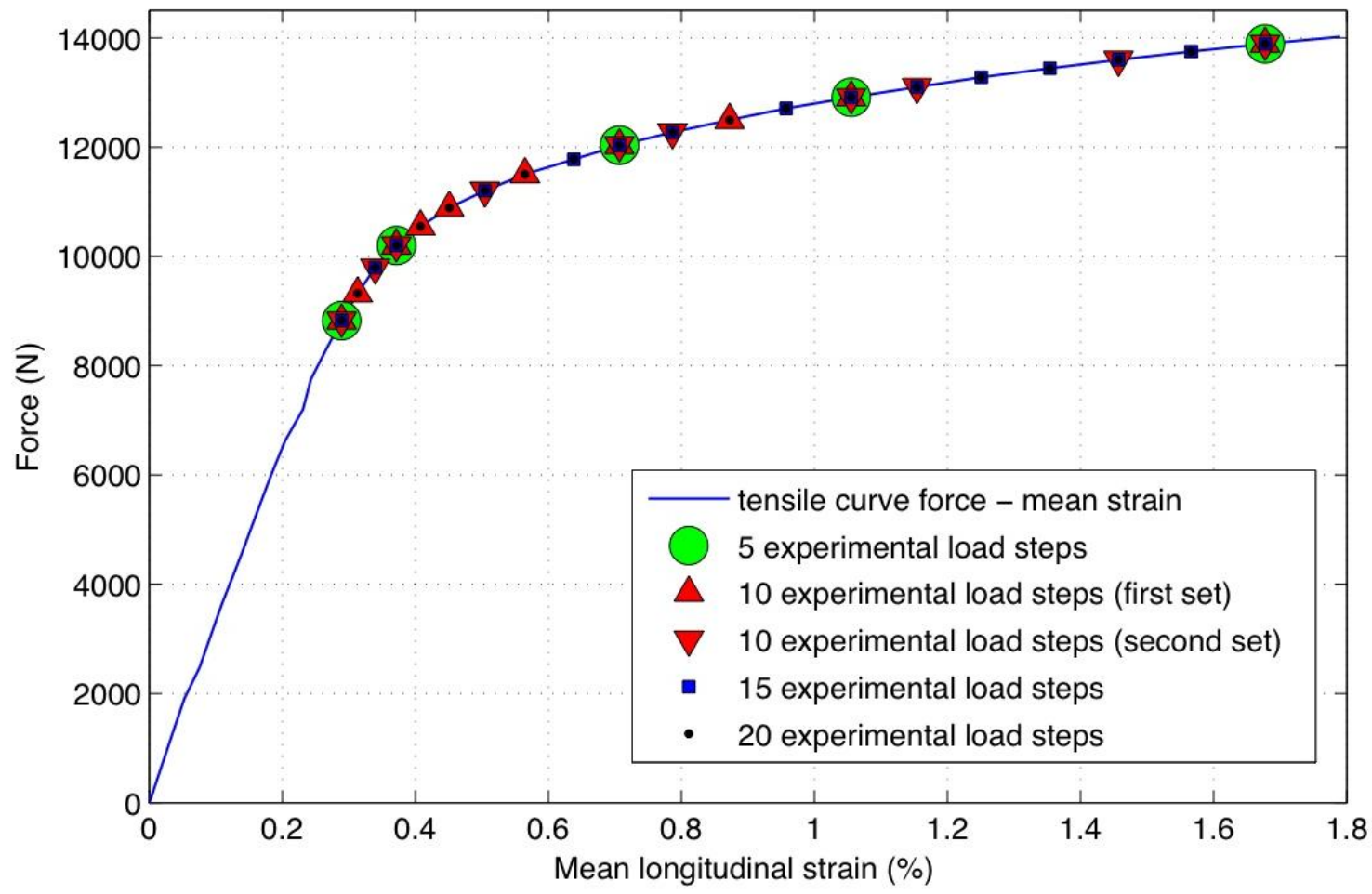

Figure 5. Experimental tensile curve (force - mean longitudinal strain) obtained for the Meuwissen specimen in solid line. The number of load steps considered in the identification procedure are superimposed in the curve (markers). 


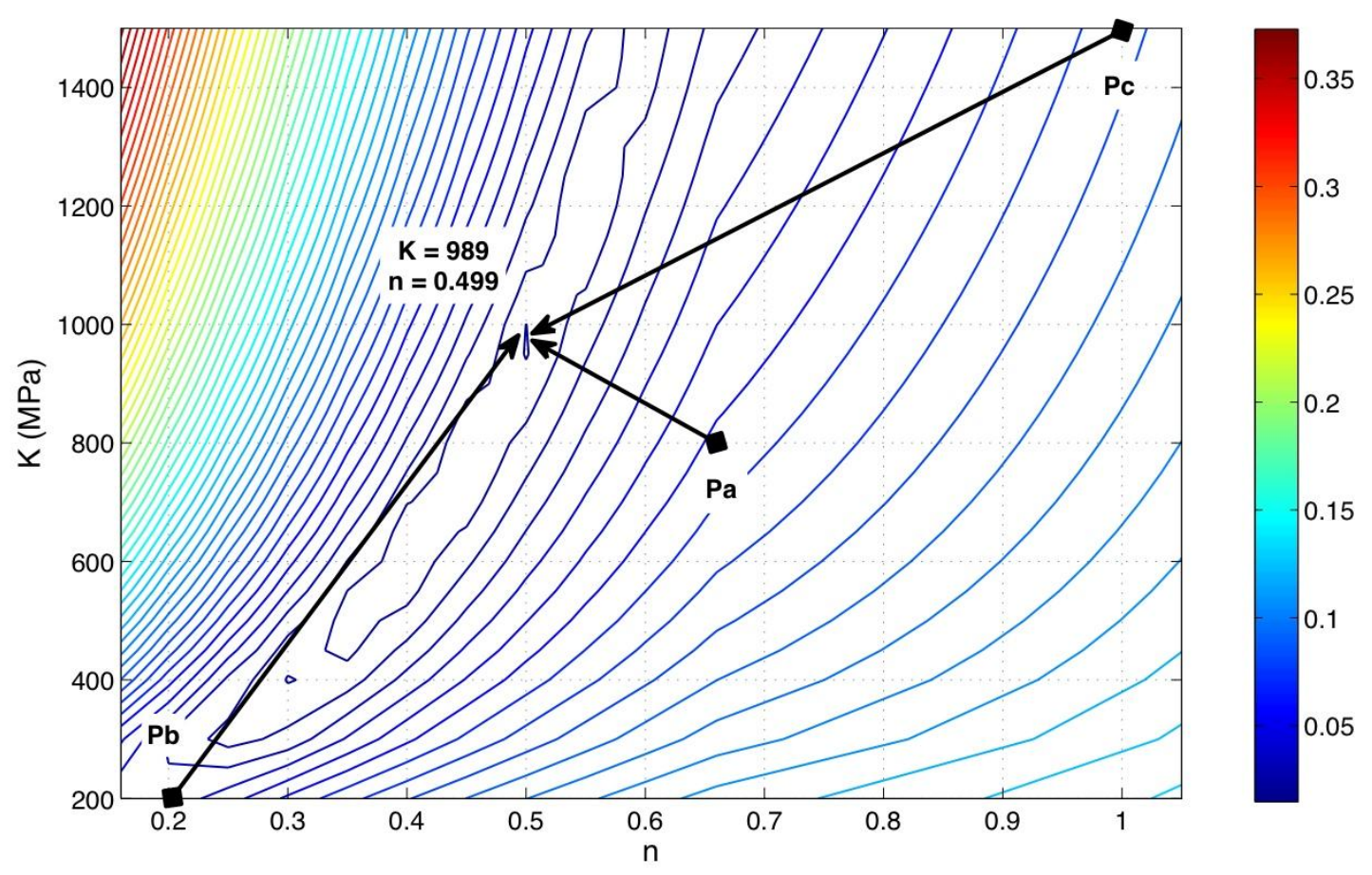

Figure 6. Sensitivity map calculated using direct FEM simulations and experimental displacement fields (20 experimental load steps) for the Meuwissen specimen geometry: overall cost function evolution versus $\mathrm{K}$ and $\mathrm{n}$. Results of the FEMU identification for the three initial parameter sets $\mathrm{Pa}, \mathrm{Pb}$ and $\mathrm{Pc}$ are superimposed in the figure (20 experimental load steps included in the identification procedure, see Fig. 5). 


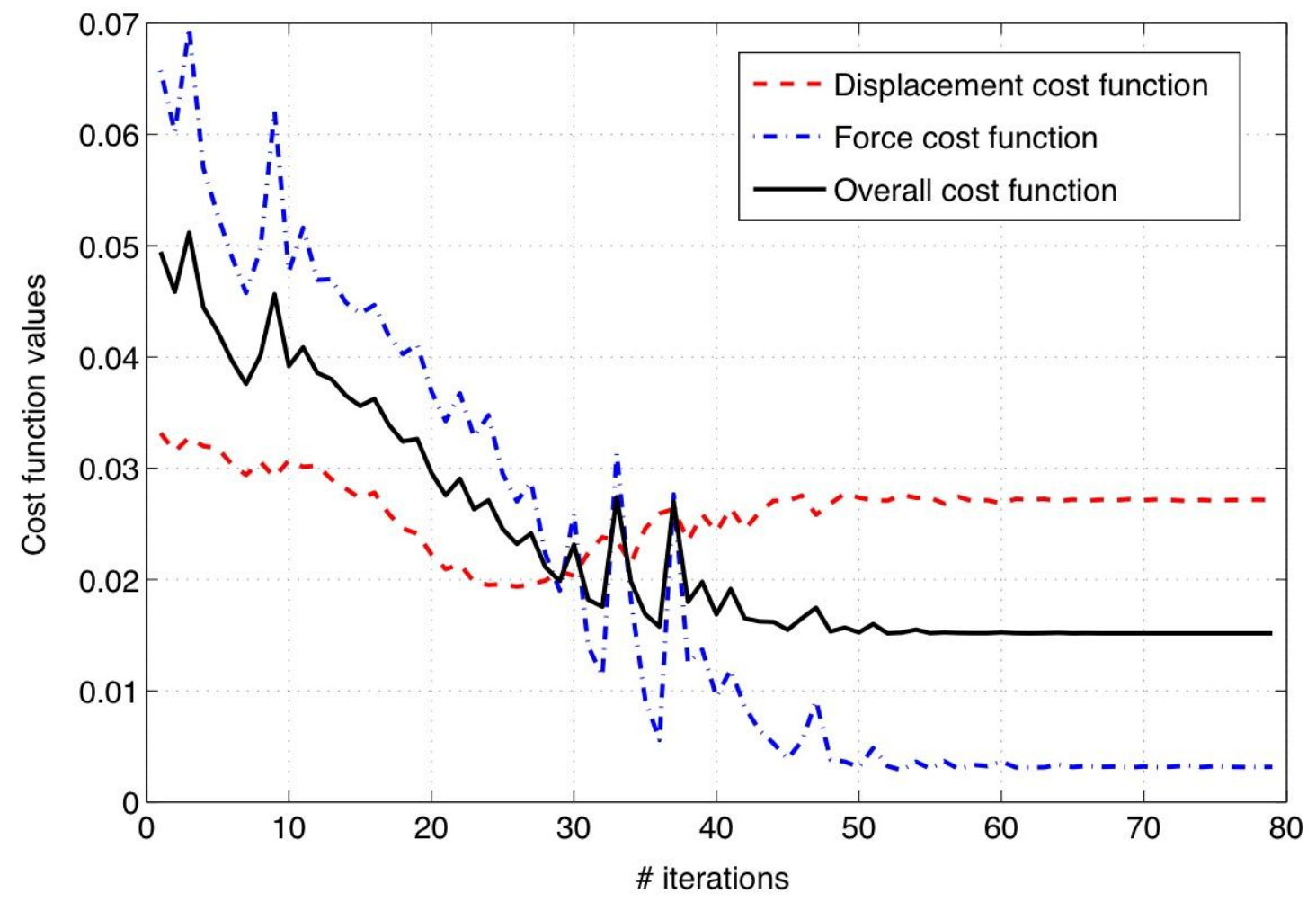

Figure 7. Evolution of the force, displacement and overall cost functions during the FEMU optimisation process (initial parameter set $\mathrm{Pb}$ ). 

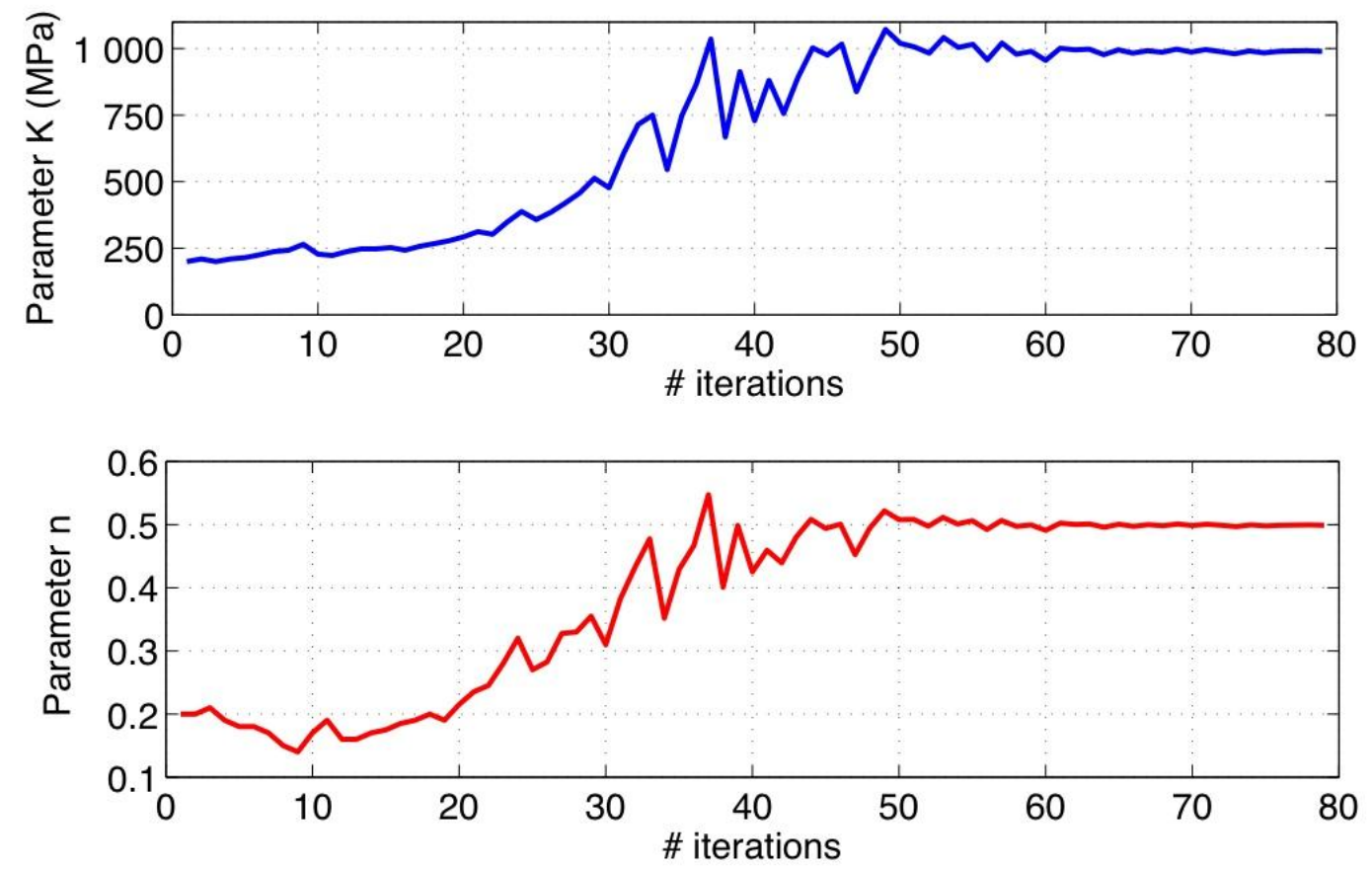

Figure 8. Evolution of the parameters $\mathrm{K}$ and $\mathrm{n}$ during the FEMU optimisation process (initial parameter set $\mathrm{Pb}$ ). 

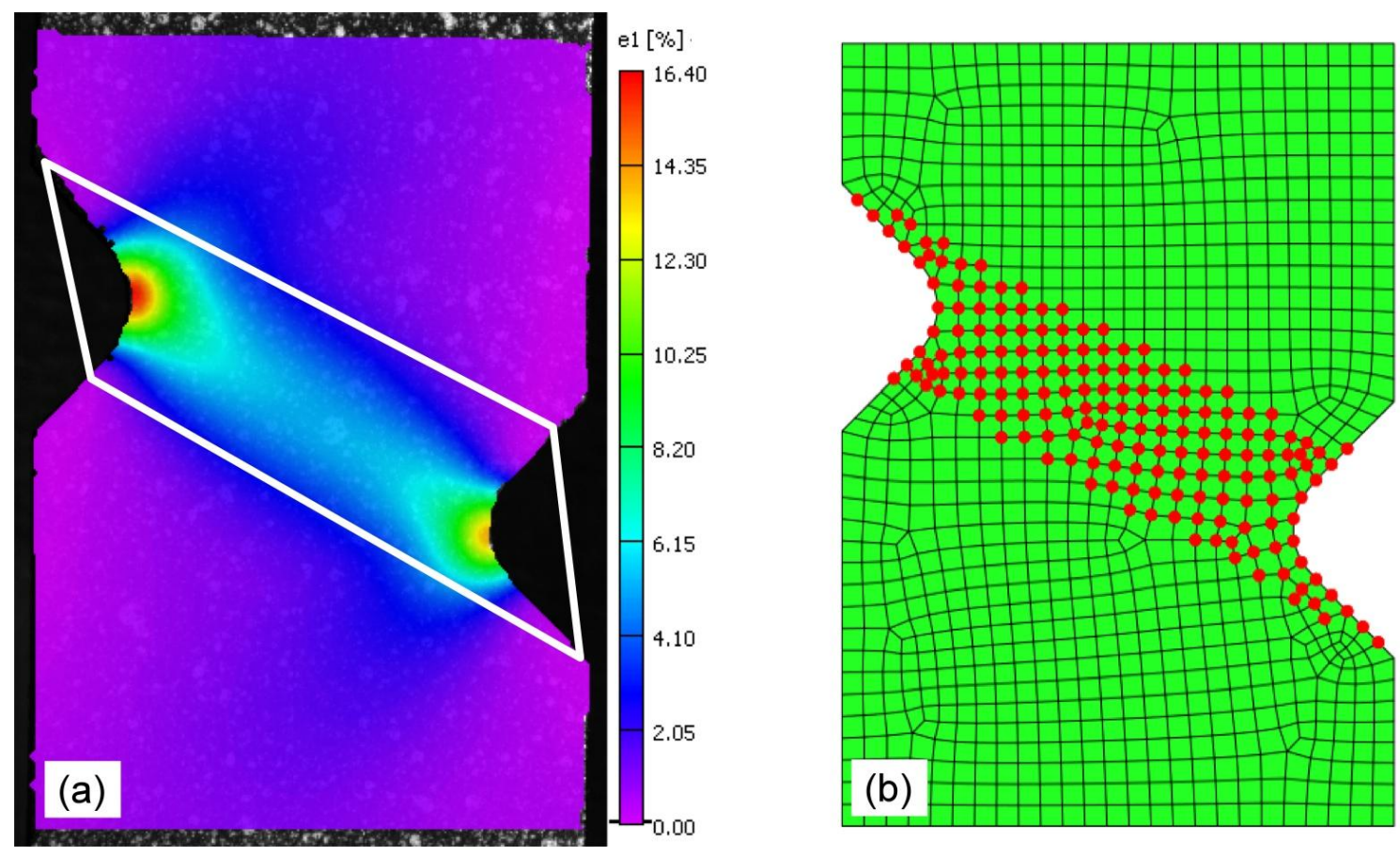

Figure 9. Meuwissen geometry. (a) Strain field (major strain) measured by DIC for the last experimental load step. The sub-area (white frame) corresponds to the area of the displacement field used for the evaluation of the displacement cost function. (b) FEM mesh. The filled points correspond to the FEM nodes used for the evaluation of the displacement cost function. 


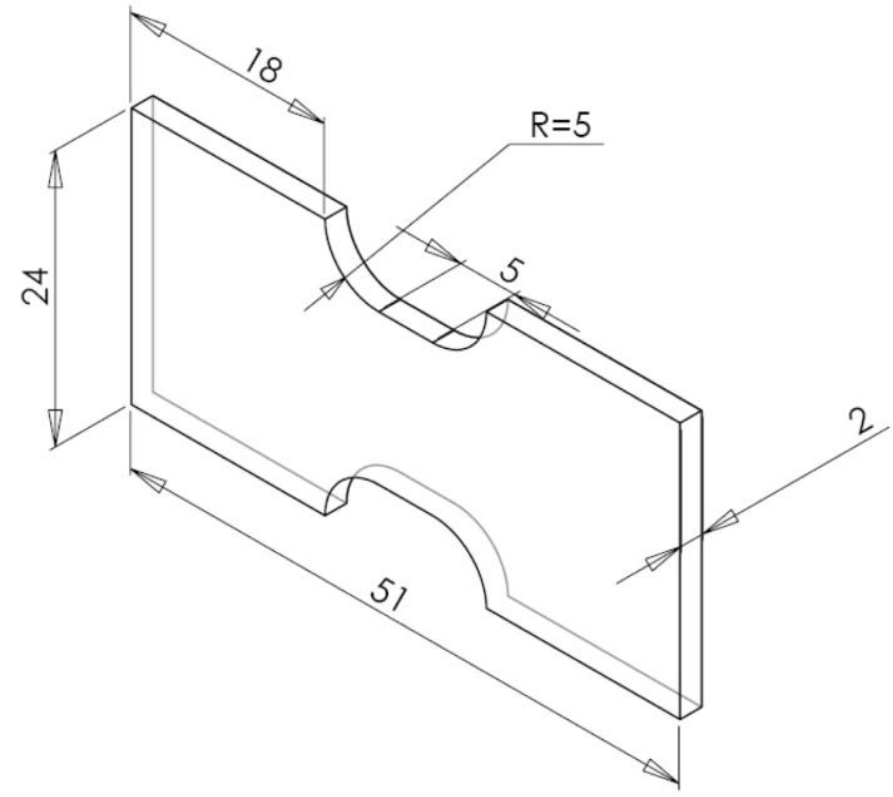

Figure 10. Geometry of the Haddadi specimen. 

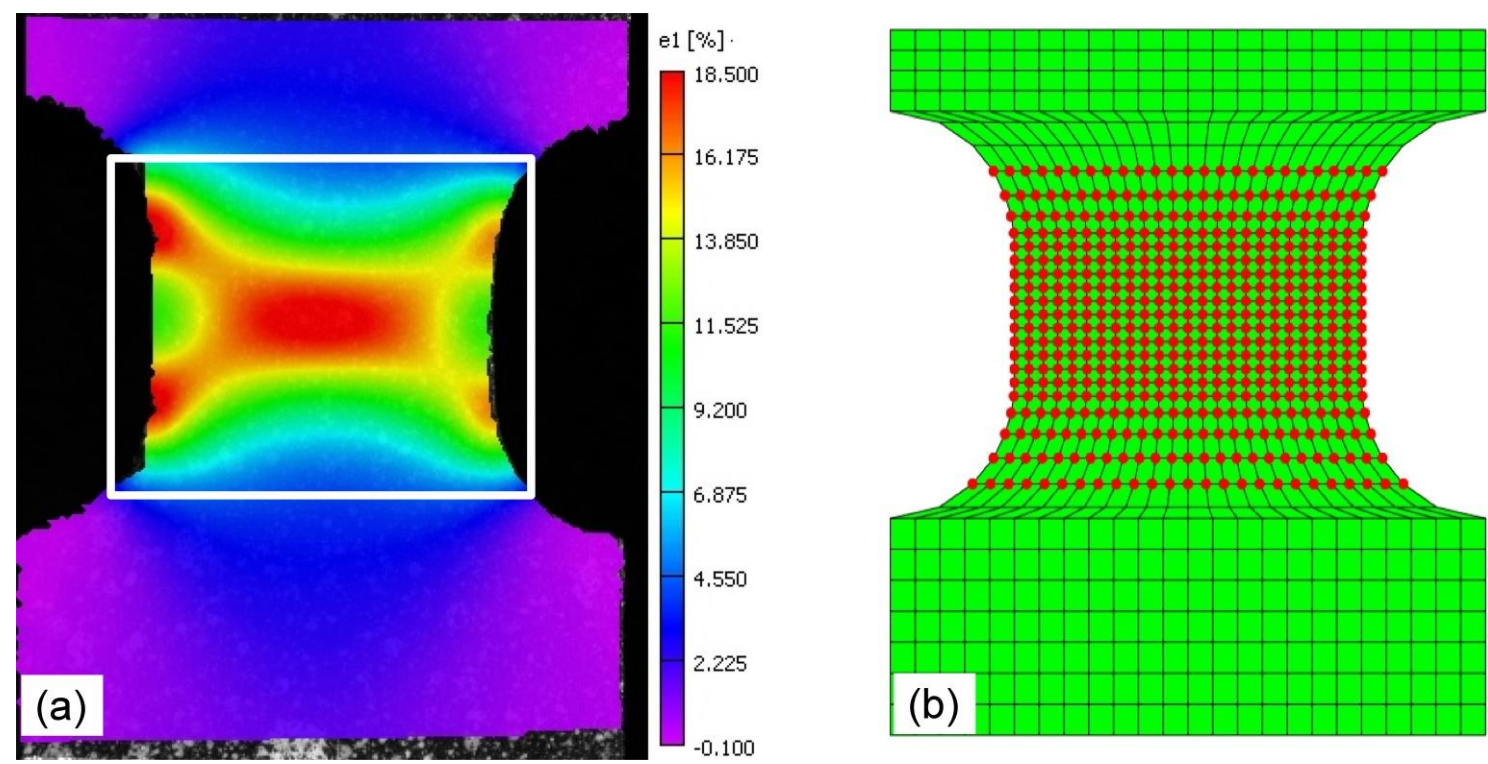

Figure 11. Haddadi geometry. (a) Strain field (major strain) measured by DIC for the last experimental load step. The sub-area (white frame) corresponds to the area of the displacement field used for the evaluation of the displacement cost function. (b) FEM mesh. The filled points correspond to the FEM nodes used for the evaluation of the displacement cost function. 


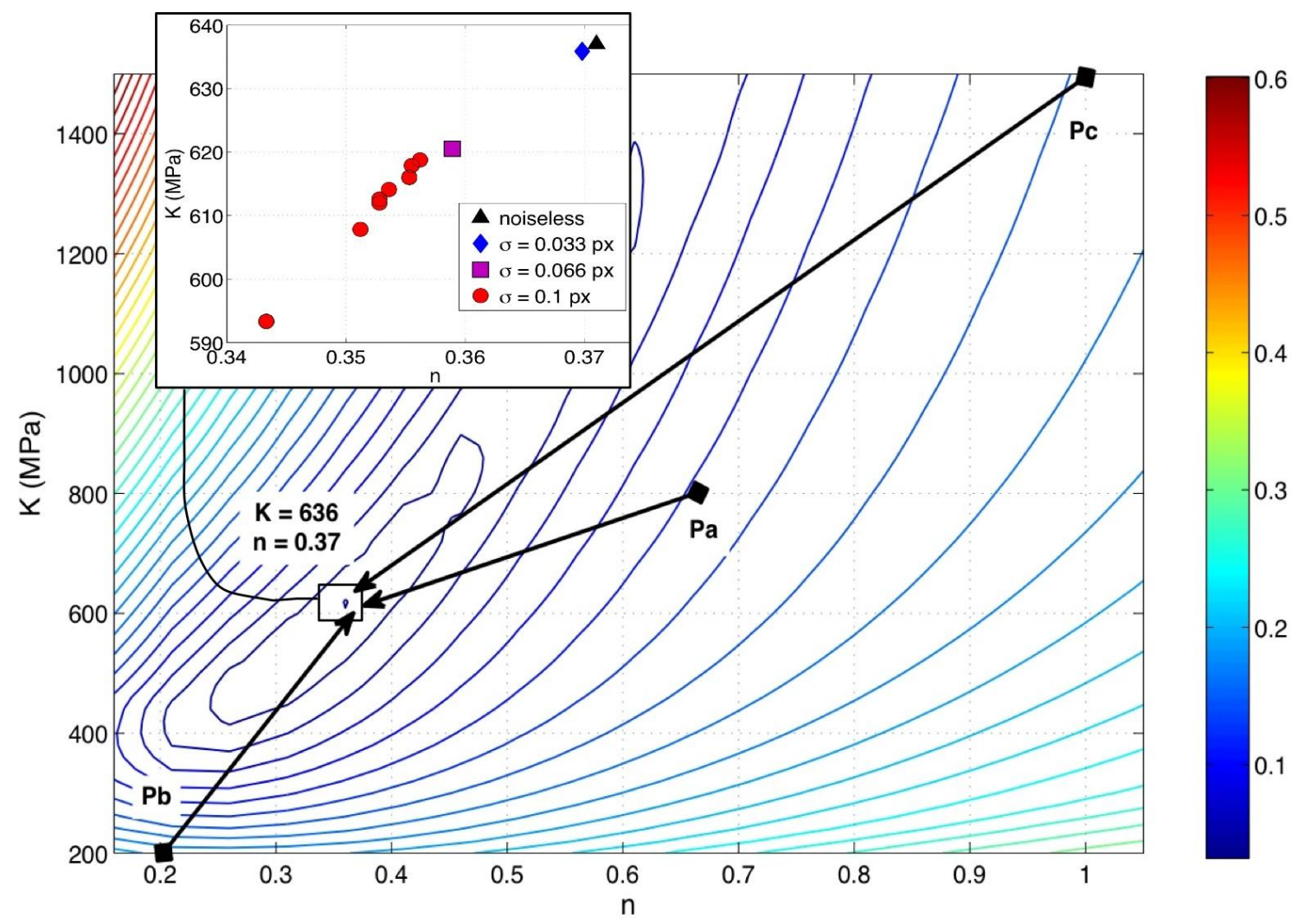

Figure 12. Sensitivity map calculated using direct FEM simulations and experimental displacement fields (ten experimental load steps) for the Haddadi specimen geometry: overall cost function evolution versus $\mathrm{K}$ and $\mathrm{n}$. Results of the FEMU identification for the three initial parameter sets $\mathrm{Pa}, \mathrm{Pb}$ and $\mathrm{Pc}$ are superimposed in the figure (ten experimental load steps included in the identification procedure). A zoom showing the effect of noisy kinematic fields is inserted. 


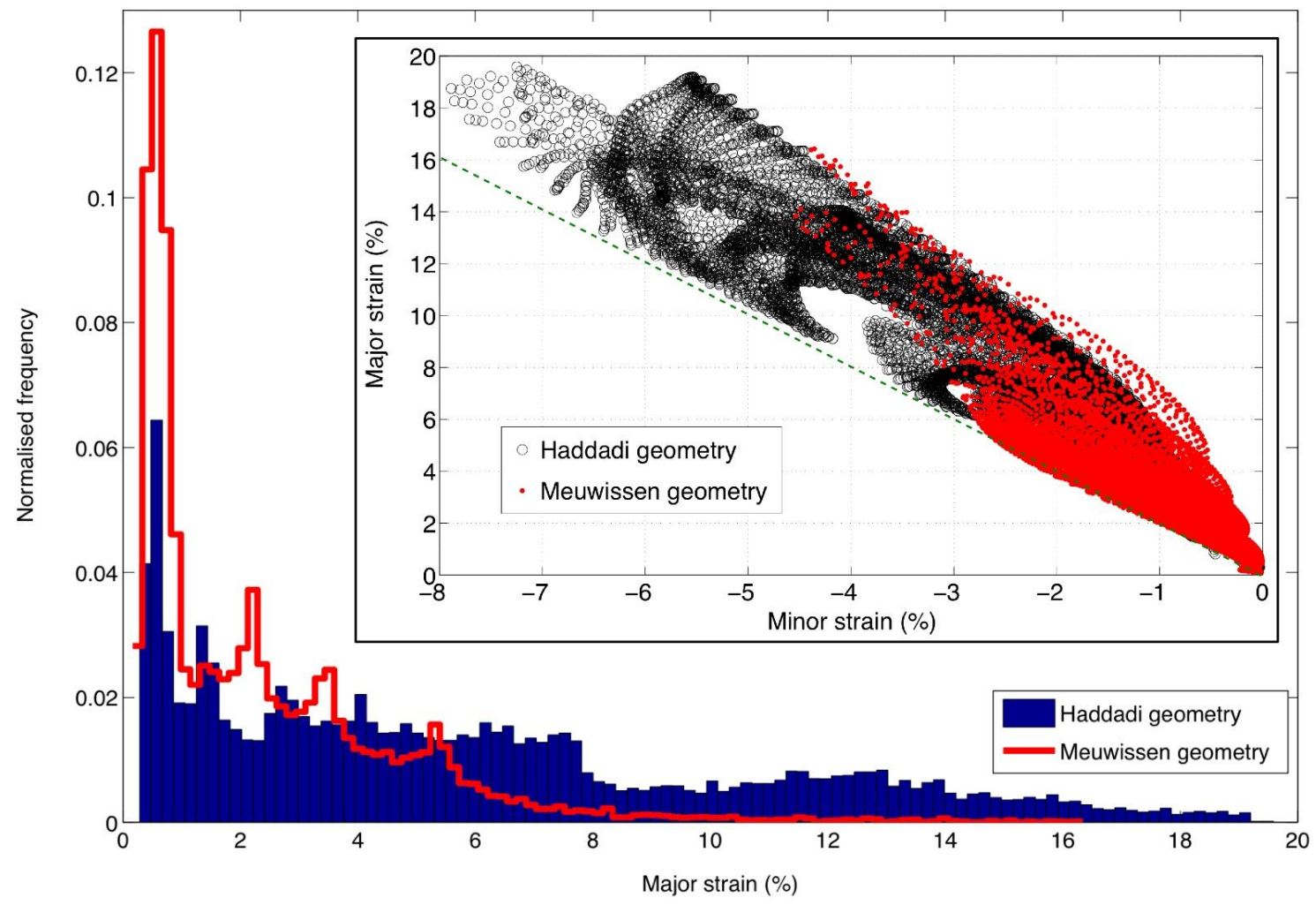

Figure 13. Histogram of the experimental major strain levels measured by DIC for both Meuwissen and Haddadi geometries. Inserted is the plot of the experimental major strain versus minor strain. Each strain fields investigated corresponds to the sum of five load steps regularly distributed between the first and the last load step. 